\title{
Overview of the role of nanotechnological innovations in the detection and treatment of solid tumors
}

This article was published in the following Dove Press journal:

International Journal of Nanomedicine

22 January 2014

Number of times this article has been viewed

\author{
Derusha Frank' \\ Charu Tyagi' \\ Lomas Tomar' \\ Yahya E Choonara' \\ Lisa C du Toit' \\ Pradeep Kumar' \\ Clement Penny ${ }^{2}$ \\ Viness Pillay' \\ 'Department of Pharmacy and \\ Pharmacology, ${ }^{2}$ Department of \\ Medical Oncology, Faculty of \\ Health Sciences, University of \\ the Witwatersrand, Johannesburg, \\ South Africa
}

\begin{abstract}
Nanotechnology, although still in its infantile stages, has the potential to revolutionize the diagnosis, treatment, and monitoring of disease progression and success of therapy for numerous diseases and conditions, not least of which is cancer. As it is a leading cause of mortality worldwide, early cancer detection, as well as safe and efficacious therapeutic intervention, will be indispensable in improving the prognosis related to cancers and overall survival rate, as well as health-related quality of life of patients diagnosed with cancer. The development of a relatively new field of nanomedicine, which combines various domains and technologies including nanotechnology, medicine, biology, pharmacology, mathematics, physics, and chemistry, has yielded different approaches to addressing these challenges. Of particular relevance in cancer, nanosystems have shown appreciable success in the realm of diagnosis and treatment. Characteristics attributable to these systems on account of the nanoscale size range allow for individualization of therapy, passive targeting, the attachment of targeting moieties for more specific targeting, minimally invasive procedures, and real-time imaging and monitoring of in vivo processes. Furthermore, incorporation into nanosystems may have the potential to reintroduce into clinical practice drugs that are no longer used because of various shortfalls, as well as aid in the registration of new, potent drugs with suboptimal pharmacokinetic profiles. Research into the development of nanosystems for cancer diagnosis and therapy is thus a rapidly emerging and viable field of study.
\end{abstract}

Keywords: nanosystems, targeted drug delivery, nanotheranostics, antineoplastic drugs, poor aqueous solubility, solid tumors

\section{Introduction}

The global burden of cancer, compounded by an increase in incidence and regression in the age of onset, has created a strong resurgence into cancer research. Large amounts of financial aid have been dedicated to research in the field of cancer etiology, detection, and treatment. However, at present, we appear to be at the losing end of the battle against cancer, which is the second leading cause of death worldwide and accounted for 7.6 million deaths in 2008. ${ }^{1}$ The burden of cancer in the African setting paints an increasingly dismal picture, with approximately 715,000 new cases of cancer diagnosed on the continent in 2008 and 542,000 deaths reported in the same period, against the backdrop of the financial burdens of this disease on the health systems of a developing continent. ${ }^{2}$ Figure 1 presents the global distribution of cancer incidence, highlighting the magnitude of cancer burden across different regions. ${ }^{3}$ The World Health Organization has projected cancer-related mortalities to rise to over 11 million by $2030 .{ }^{1}$
Correspondence: Viness Pillay

Department of Pharmacy and

Pharmacology, Faculty of Health

Sciences, University of the

Witwatersrand, 7 York Road, Parktown,

Johannesburg 2193, South Africa

Tel +27 II 7172274

Fax +27 I I 6424355

Email viness.pillay@wits.ac.za
International Journal of Nanomedicine 2014:9 589-6/3 


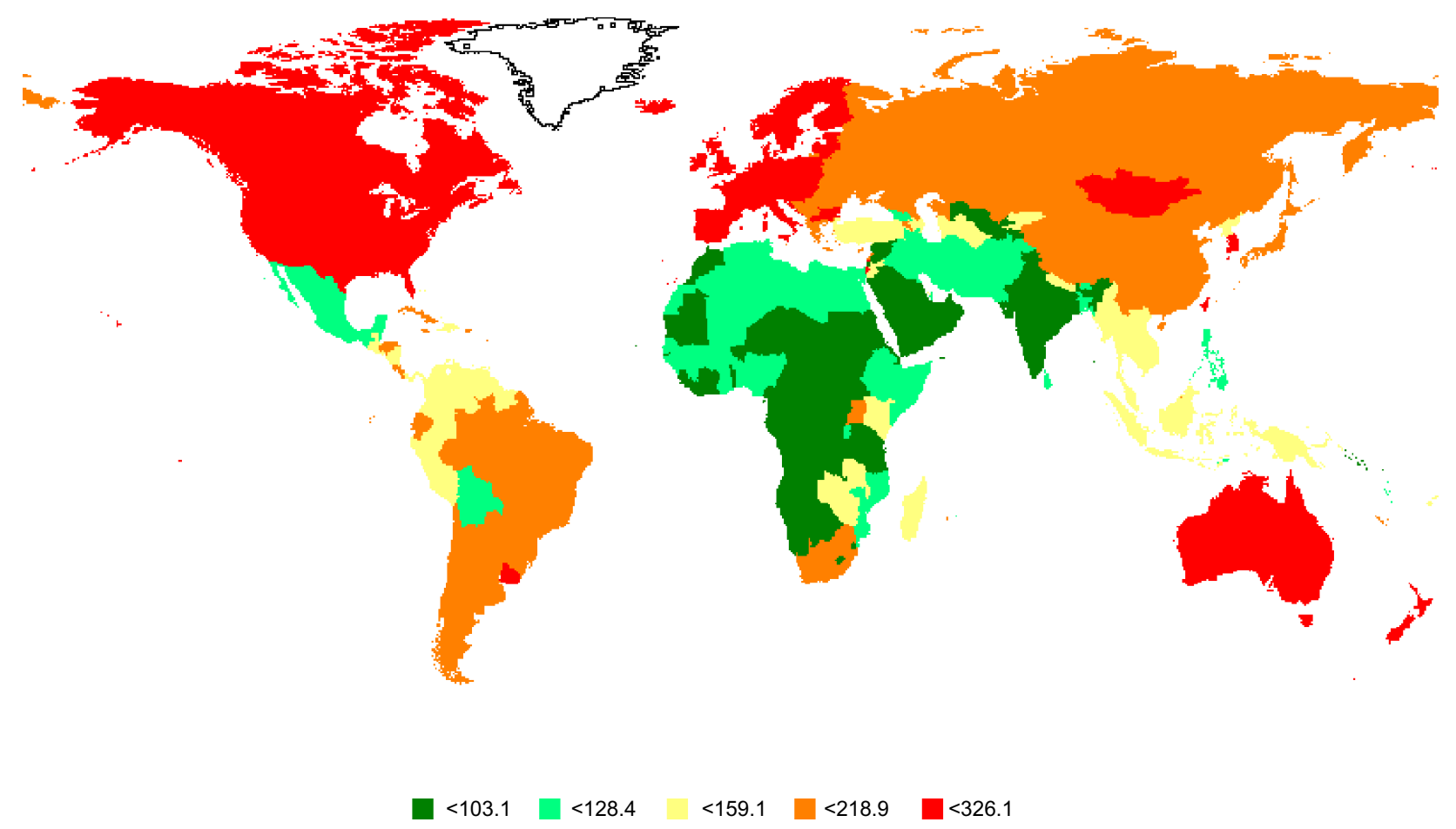

Figure I Estimated age-standardized incidence rate (worldwide) per 100,000 population. ${ }^{3}$

Note: *Region estimates do not sum to the worldwide estimate due to the calculation method.

Cancer, as defined by the National Cancer Institute in the United States, is an uncontrollable division and proliferation of abnormal cells and the invasion of these cells into the surrounding tissue. ${ }^{4}$ This is a generic term and encompasses a multitude of malignancies that can affect numerous bodily systems. Carcinogenesis is a multistep process, involving distorted expression of the transcriptional factors involved in cell replication. ${ }^{5}$ The accumulation of sufficient alterations leads to mutations in the genes regulating cellular growth and differentiation and inhibits programmed cell death. Genetic alteration may further lead to the activation of oncogenes and deactivation of tumor suppressor genes. The cumulative effect is malignant transformation of cells, rapid growth and proliferation of these malignant cells, amplified angiogenesis, invasion into organs and body cavities, and subsequent metastasis. Metastasis refers to the spread of the cancerous cells from the point of origin to other body parts and systems through the vascular and lymphatic systems.

Nanomedicine refers to the research and development of technologies, devices, and drug delivery systems for prevention, diagnosis, and treatment of disease at the nanoscale. ${ }^{6,7}$ Nanostructures, as per the definition, are varying geometrical configurations with at least one dimension in the range of 1 to $100 \mathrm{~nm}$. However, nanostructures up to $250 \mathrm{~nm}$ in size have found applicability in the medical setting by the enhanced permeability and retention (EPR) effect characterizing certain pathologies, by active targeting, enhanced residence time, and increased cellular uptake. ${ }^{8,9}$ Nanosystems combine the fields of medicine, chemistry, pharmacology, biology, mathematics, and engineering to impart unique properties that are yet to be fully explored. ${ }^{7,10}$ Nanosystems with biomedical application are currently a highly researched field and have exhibited immense potential, particularly in the diagnostic, imaging, and therapeutic domains. ${ }^{11}$ Numerous benefits of nanosystems are related to the augmented surface area-tovolume ratio, which has the potential to favorably influence the solubility, bioavailability, pharmacokinetic, and biodistribution profile of drugs, as well as, their inclination toward modification and functionalization. ${ }^{6,12-14}$

Nanomaterials have been shown to exhibit properties vastly different from the bulk material from which they are synthesized. ${ }^{12,15,16}$ In addition, the vast array of structural arrangements make nanosystems incredibly versatile, whereas their minute dimensions often belie an astonishing robustness. These devices offer the unequivocal advantage of interacting with cells, biological processes, and other targets at a molecular level, allowing for inter- and intracellular manipulation and monitoring, the benefits of which are beyond our full comprehension at this point. ${ }^{17}$ The continued development of novel nanosystems is expected to 
have extensive applicability at all levels of health care, including disease initiation, progression, treatment, and monitoring. Nanosystems are expected to enable superior detection of molecular changes that precede pathogenesis, thereby enabling early detection, proactive treatment, and monitoring, as well as expanding the knowledge base on mechanisms of disease progression. These systems have the potential to enhance selectivity and sensitivity for pathological states to advance disease diagnosis and imaging and transform drug delivery and therapy by capitalizing on the unique properties of the nanoscale size range. ${ }^{10}$ The design of multifunctional nanosystems aims at and is expected to effectively combine diagnostic, therapeutic, and efficacy monitoring applications in one unit. ${ }^{10}$ Finally, and perhaps of greatest significance, nanotechnology and nanosystems will contribute to, and perhaps transform, the scientific approaches to all of the above as well as the processes involved in drug discovery, purification, and modification.

Research and development into nanosystems applicable to cancer diagnosis, imaging, and therapy has emerged as one of the largest fields in nanomedicine. The substantial burden of cancer on the global population and the indiscriminate nature of the condition underscore the considerable interest of governmental, industrial, health care, and academic entities in exploring and developing the applicability of this technology to the oncology field. This review aims to discuss the exciting role and immense potential of nanotechnology in the diagnosis, imaging, and treatment of solid tumors. For the purpose of this review, the terms "nanosystem" and "nano-enabled drug delivery/imaging system" shall be used interchangeably and will encompass nanoparticles, micelles, liposomes, quantum dots (QDs), nanotubes, and all other drug delivery and imaging systems in which at least one dimension is of the nanoscale.

\section{Nanotechnology in tumor diagnostics and imaging}

Early diagnosis and precise imaging of carcinogenesis is critical to successful therapeutic outcomes. However, highly sensitive and specific technologies are required to detect slight biological changes that may characterize early-stage disease, as well as to accurately classify the stage and dissemination of tumor. One of the major setbacks in the early detection of tumors of the ovary, pancreas, and liver is a lack of suitable detection technologies and imaging techniques. Accurate imaging techniques will assist in the early diagnosis of these tumors, accurate staging of the tumor, as well as in monitoring the efficacy of treatment and early determination of resistance to the therapy being used. Recurrence, along with the emergence of resistance, to antineoplastic drugs is an enormous problem in ovarian cancer, resulting in atrocious 5-year survival rates. However, when ovarian cancer is diagnosed in the early stages (when it is confined to the ovary), the 5-year survival rate is approximately $90 \%{ }^{18}$

Current systems employed for the detection of tumor cells or cancer biomarkers include surface plasmon resonance, nuclear magnetic resonance, quartz crystal microbalance resonators, and polymerase chain reaction, among others. These systems call for augmentation of the signal, the expression of fluorescent protein biomarkers and antibodies in the cell, or the enhancement of target cells in the sample. In view of this, a detection system that is sensitive, minimally invasive, and not requiring augmentation or labeling of the sample would be highly beneficial. ${ }^{19}$ Nanoscale detection and imaging devices have the capacity to demonstrate substantial sensitivity and specificity, which could revolutionize the clinical course of cancers. The nanoscale offers the advantage of interacting and detecting changes at intra- and intercellular levels and is thus unsurpassed in analyzing most biological moieties such as cells, viruses, proteins, and nucleic acids. $^{12}$

\section{Nanostructure-based biosensors in cancer detection}

Biosensors consist of a bioreceptor that interacts with specific analytes within the body, producing a change that is detectable through conversion by a transducer (constituent of biosensor) to a measurable signal. ${ }^{20}$ Figure 2 outlines the interactions that are responsible for changes that occur and the types of signals that are measured after transduction.

Nanostructures, particularly nanofibers and nanoparticles, have been applied to the field of biosensors, creating nanobiosensors. ${ }^{20}$ The initial application of nanofibers to biosensors was an antibody-based system for the detection of benzo[a] pyrene tetrol in the body, which indicates exposure to benzo[a]pyrene $(\mathrm{BaP}) .{ }^{20} \mathrm{BaP}$, a common environmental pollutant as well as a component of tobacco smoke, is a known human carcinogen. ${ }^{19,20}$ Early detection of exposure to $\mathrm{BaP}$ can have remarkable implications in the detection and treatment of, in particular, lung cancer, which is a leading cause of cancer death worldwide. ${ }^{19}$

$\mathrm{Wu}$ et al combined biocompatible and biodegradable poly(lactic acid) nanofibers with gold nanoparticles, which 


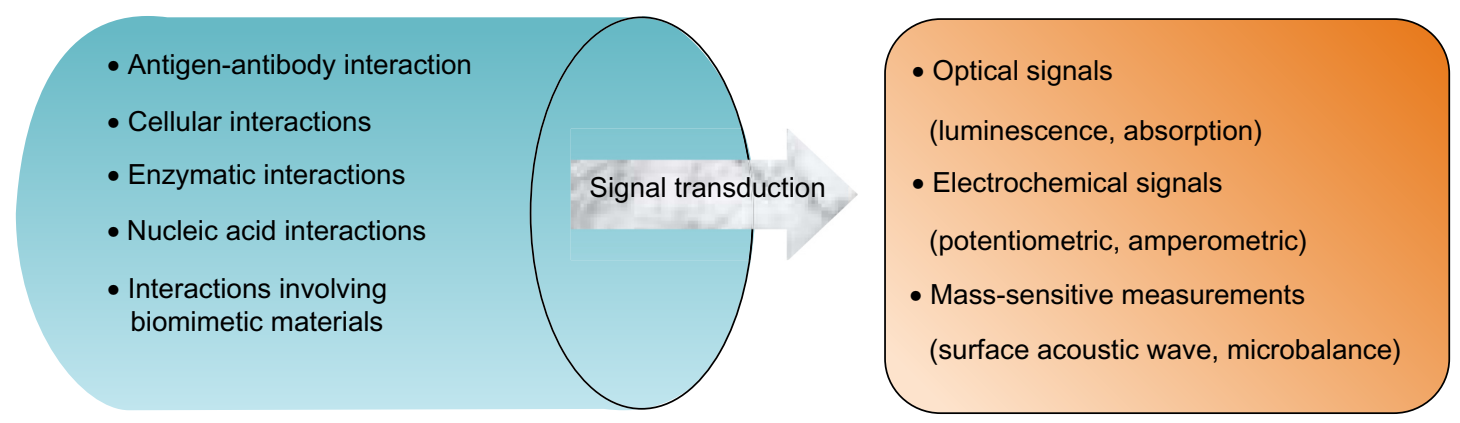

Figure 2 Illustrative representation of the various types of interactions between bioreceptors and analytes, as well as the measurable signals that are produced.

have been applied extensively in the field of biosensors, to form nanocomposites. ${ }^{19}$ These nanocomposites were subsequently used on modified indium tin oxide electrodes and investigated for their ability to detect drug-sensitive and drug-resistant leukemia cells. These gold/poly(lactic acid) nanocomposite modified indium tin oxide electrodes exhibited promising sensitivity in the detection of leukemia cells, as well as differentiating between the cell types. ${ }^{19}$ This nano-biosensor has potential for the rapid and early detection of cancer cells, which will be of considerable value in clinical practice.

Angiogenesis, the process whereby new blood vessels are developed in the human body, is essential to the growth and preservation of living tissue. ${ }^{21}$ However, this process occurs at an elevated rate in tumor tissue and is related to proliferation and metastasis of cancer cells. ${ }^{21}$ Vascular endothelial growth factor (VEGF) is a potent proangiogenic agent that is pivotal to the angiogenic process, and thus an attractive target for cancer therapy, as well as a useful means for early cancer detection..$^{21}$ Current methods for the detection and quantification of VEGF include enzyme-linked immunosorbent assay, VEGF receptors, and field-effect transistor biosensors. ${ }^{21}$ There, however, exists room for improvement in the sensitivity and ease of use of these tests. To this end, Kwon et al combined conducting polymer (polypyrrole) nanotubes with aptamers (nanosize molecules with a great degree of sensitivity and specificity for target molecules), bonded to the nanotubes, in a modified biosensor for the detection of VEGF. ${ }^{21}$ Two carboxylated polypyrrole carbon nanotubes (CPNT) were synthesized (CPNT 1, 190-220 nm; CPNT 2, 100-130 nm), attached to biosensors, and evaluated. Although both CPNTs displayed a high degree of sensitivity in detecting VEGF, CPNT 2 exhibited approximately twofold higher sensitivity, highlighting the significance of size in this detection method. The increased sensitivity of the transducer with CPNT 2 was attributed to increased conductivity as a result of the augmented surface area exposed in CPNT 2, a consequence of the smaller dimensions. ${ }^{21}$ The presence of aptamer on CPNT 2 produced superior sensitivity compared with non-aptamer based biosensors and the larger size of CPNT 1. In addition, rapid real-time detection adds another favorable dimension to this system, as the biological events detected can be converted directly into a measurable signal and no labeling of samples is required. ${ }^{12,21} \mathrm{Kwon}$ et al were successful in detecting previously unprecedented low levels of VEGF, which could have enormous implications for the early detection of several cancers. Furthermore, the modified biosensor system developed is reusable, enhancing the practicality and attractiveness of this detection device. ${ }^{21}$

In the case of colorectal cancer, doctors examine the colorectal lining with the aid of a fiberoptic camera; this screening process is called colonoscopy and allows for the detection of small growths in the colorectal tissue, which are removed. Researchers in this field strive to improve the accuracy of colonoscopy to detect the colorectal cancer even earlier. In one approach, the Center for Cancer Nanotechnology Excellence at Stanford University, part of the National Cancer Institute's Alliance for Nanotechnology in Cancer, has developed a system using gold nanoparticles that identify and bind to cancer cells. The light thrown or shined over the colorectal tissue by means of a device inserted within the standard endoscope (such as a colonoscope) makes cancer cells bound to nanoparticles stand out from the normal cells, and thus, able to be removed. Researchers plan to test the safety of this approach in humans in clinical trials. ${ }^{22}$

Telomerase is regarded as a general biomarker for cancer because of a significant upregulation ( $85 \%-90 \%)$ in its expression in human tumor cells. The detection of this biomarker, thus, has enormous potential in early cancer detection, as well as monitoring the efficacy of chemotherapeutic interventions. ${ }^{23}$ Telomerase is located in trace amounts within cell nuclei, and therefore the ideal 
detection of telomerase will involve ultrasensitive single cell analysis of living cells. Nanofiber biosensors conjugated with antitelomerase antibody are capable of intracellular detection of telomerase because of its size and the direct interaction between conjugated antitelomerase and telomerase by penetration of the cell nucleus. ${ }^{23}$ The significance of the nanoscale was once again highlighted in this study, whereby detection of telomerase was attempted by employing the cytoplasm of cancer cells. There was no significant detection of telomerase, emphasizing the importance of nucleus penetration for telomerase detection. ${ }^{23}$ This would be impossible with larger devices. Furthermore, fluorescence was significantly reduced when cells expressing low levels of telomerase were assayed. The nanofiber biosensor is thus able to differentiate normal cells from telomerase overexpressing cancer cells, making telomerase detection by nanosystems a superior detection technique.

\section{QDs for tumor detection and imaging}

QDs are semiconductor, fluorescent nanocrystals that have received considerable attention in the biomedical field, particularly for their potential as imaging agents. ${ }^{24-26}$ The advantages of QDs over the conventional dyes employed for imaging include tailoring of the spectrum of emission by alteration of the size and composition of QDs, the high quantum yield of fluorescence and superior brightness exhibited by QDs, a broader spectrum of excitation, and more rapid imaging. ${ }^{24-28}$ Moreover, QDs demonstrate a high degree of photostability and resistance to photobleaching, which is imperative for in vivo application..$^{24-27}$

However, the use of QDs has been restricted, as a consequence of toxicity from semiconductor QDs (particularly cadmium-based QDs) and inadequate in vivo and physical stability profiles, partly because of their hydrophobic nature. ${ }^{24,26,29}$ Modifications of QDs have therefore been attempted in the form of surface coating and entrapping QDs in water-soluble nanoparticles and lipid micelles. ${ }^{24}$ These methods have shown potential in increasing the stability of QDs, as well as improving the biocompatibility and biodistribution and reducing toxicity. ${ }^{26}$ As outlined with previously discussed nanosystems, biocompatible and stable QDs can then be functionalized for selectivity and site-specific targeting. ${ }^{30}$ This will prove beneficial in the early detection and diagnosis of cancer, which forms the basis of successful therapeutic interventions. Furthermore, QDs have shown promise in the detection of sentinel lymph nodes, which is exceptionally beneficial in rapidly metastasizing cancers.
Encapsulation of QDs in lipid micelles has been investigated as a means of addressing the drawback of instability, as well as investigating the efficacy of targeting, by further functionalization of these micelles. ${ }^{25}$ The aforementioned preparation proved nontoxic to cells when compared with cells incubated in the absence of the formulation and cells that were incubated with a nontargeted formulation, as well as highly selective for target cells $(80 \%-90 \%) .{ }^{25}$ As a practical consideration, entrapped QDs displayed impressive stability during a prolonged period, with preservation of fluorescent capacity, making them viable for clinical use. Analysis by Schroeder et al of pegylated lipid micelles entrapping QDs 1 year after preparation displayed no difference in structure, optical properties, and physical stability (aggregation or precipitation).$^{25}$ This favorable stability profile was demonstrated in other studies for shorter periods (3 months). ${ }^{27}$ Differing results on fluorescence intensity were reported by Schroeder et al and Papagiannaros et al after encapsulation of QDs in micelles. ${ }^{25,27}$ The first group observed a decrease in fluorescence intensity of encapsulated QDs compared with single QDs, which was attributed to a quenching effect by adjacent QDs. However, the stability of the formulation acted to counter this effect, and ultimately the fluorescent capacity was sufficient for the intended in vivo application. ${ }^{25}$ In contrast, the second group reported a greater intensity in fluorescence of QD-encapsulating-micelles. ${ }^{27}$ Despite this disparity, both research groups demonstrated clear and precise tumor imaging. Hence the systems described by Schroeder et al and Papagiannaros et al addressed many of the in vivo shortfalls established by previous work on QDs and served to further highlight the feasibility of this nanosystem in the domain of cancer detection and imaging. ${ }^{25,27}$

The nanoscale of QDs is essential to the imaging function, as it affects biodistribution. Larger QDs may be confined to the blood vessels, thereby producing diffuse fluorescence and poorly differentiated tumor tissue and organs. In contrast, the nano size will allow extravasation from blood vessels and accumulation of the QDs in tumor tissue, resulting in clearly contrasted and delineated tumoral images with high-intensity fluorescence and reduced background noise. ${ }^{27}$ Furthermore, differential QD accumulation in organs has the potential to highlight distant metastasis.

In the domain of cancer, imaging techniques are used to stage tumors as well as to aid surgeons undertaking cytoreductive surgery. For this application, emission spectra should be between 400 and $650 \mathrm{~nm}$ to enable visibility without the use of specialized equipment. ${ }^{30}$ At this time, imaging in 
the near-infrared region is widely used, as it is a minimally invasive process that enables clear, detailed imaging at a molecular level. ${ }^{27}$ However, tissue penetration is limited. This technique has been applied in tumor imaging, as well as mapping of tumor biomarkers and sentinel lymph nodes, as an aid in surgical resection. Furthermore, this technique has been used to monitor the response of tumors to chemotherapy. ${ }^{27}$ The emission spectrum of nanosystems, such as QDs, can be tailored for specific applications. However, the imaging of tumors and metastatic tissue requires sufficient tissue penetration, which usually occurs at emission wavelengths that are out of the visible spectrum. Therefore, modifications are required to satisfy the two requirements pivotal to successful tumor and metastatic tissue imaging.

Multispectral imaging is an emerging technique that involves the splitting of signals for the detection of different moieties by a single excitation. ${ }^{30}$ This will allow simultaneous superficial as well as deep tissue imaging while minimizing the photodamage of surrounding tissue. ${ }^{24,30}$ Indium phosphide/zinc sulfide QDs have been formulated with an exciton emission and a defect emission that allows for simultaneous superficial imaging and deep tissue penetration. ${ }^{30}$

\section{Nanotechnological approaches to cancer therapy}

Challenges to successful antineoplastic therapy are severalfold. First, poor aqueous solubility poses significant formulatory and delivery challenges. Second, the physiological environment presents barriers to high drug bioavailability. Serum proteins can attach to drug molecules, rendering them unavailable for absorption and cellular internalization to exert their antineoplastic activity. Physiological instability, metabolic degradation, and renal clearance may further compromise drug concentration at the site of action. However, it is the indiscriminate activity of antineoplastic drugs and the resultant adverse effects that portray possibly the greatest challenge. These adverse effects can be dose-limiting, thereby compromising therapeutic efficacy and outcome, or may themselves prove fatal.

The potential of nanosystems in addressing and overcoming the aforementioned drawbacks associated with current antineoplastic therapy reside in their ability to, first, encapsulate lipophilic drugs within the nanosystem or employ a nanovehicle to successfully deliver these drugs in vivo. ${ }^{31}$ Formulation as nanosystems, particularly encapsulation within a nanosystem, serves a protective function from inactivation and metabolic degradation. ${ }^{31}$
Second, nanosystems smaller than $300 \mathrm{~nm}$ are able to circumvent the reticuloendothelial system, thereby increasing the circulation half-life and improving the pharmacokinetic profile of antineoplastic drugs. Third, one of the foremost benefits in the application of nanomedicine to cancer therapeutics lies in the passive targeting and accumulation attributes of nanosystems to tumor tissue as a result of the EPR phenomenon displayed by tumor tissue. In addition, the large surface-area-to-volume ratio of nanosystems facilitates the attachment of targeting moieties for active targeting to tumor sites. Active and passive targeting favorably alter the biodistribution of antineoplastic drugs, simultaneously reducing adverse effects and increasing the concentration of drug within tumor tissue for the augmentation of therapeutic antineoplastic effect. ${ }^{31,32}$ Finally, formulation as nanosystems have enabled direct uptake of the nanosystems into tumor cells by endocytic pathways and bypassing of the cellular efflux pump, thereby overcoming a mechanism of drug resistance. ${ }^{31,33}$

The potential of nanotechnological engineering and manipulations in addressing the various challenges limiting the success of antineoplastic therapy will herein be addressed with reference to poor aqueous solubility of antineoplastic drugs, passive and active targeting of nanosystems in enhancing therapeutic efficacy, and the increasing problem of emergent drug resistance.

\section{Nanosystems for improving aqueous solubility of drugs}

Considering that water constitutes more than $50 \%$ of the human body, administered drugs are bound to encounter an aqueous environment on their path to the site/s of interest. ${ }^{34}$ Furthermore, most routes of administration, particularly intravenous administration, require solubilization in an aqueous medium. However, the problem of administering poorly aqueous soluble drugs continues to plague scientists. Approximately $40 \%$ of new chemical entities are poorly aqueous soluble or aqueous insoluble, hindering formulation development and the use of effective drug molecules. ${ }^{35-37}$ Moreover, new drug development has been stifled because of unsatisfactory biopharmaceutical properties, not least of which being problems related to aqueous solubility. ${ }^{38,39}$ Poor aqueous solubility is one of the fundamental problems of antineoplastic drugs and has thus been the stimulus for the development of carrier systems for these drug molecules, among other modifications.

The aforementioned augmented surface area to volume ratio exhibited by nanosystems has drastic effects 
on aqueous solubility. ${ }^{39,40}$ This approach has been widely exploited, particularly in the realm of drug administration in cancer therapeutics. In addition to improving aqueous solubility of antineoplastic drug molecules, the nano size range allows for more rapid dissolution of the drug within the body, allowing for easier absorption and greater propensity to exert its effect. ${ }^{39,40}$ These favorable effects of nanosizing can be succinctly explained by the Noyes-Whitney and OstwaldFreundlich principles, which associate the nanoscale size range to an amplified particle dissolution velocity as well as saturation solubility, ultimately favoring increased drug bioavailability..$^{40,41}$

\section{Nanodrug delivery systems for targeted cancer therapy}

The idea of targeted therapy was first postulated by Paul Ehrlich (1906), with the objective being the attainment of therapeutically effective doses while preserving the state of healthy tissue. ${ }^{42-45}$ As previously highlighted, the path to effective drug delivery to tumor tissue and successful treatment outcomes is marred by a multitude of factors, not least of which is compromised bioaccessibility, and thereby poor bioavailability of antineoplastic drugs at tumor sites. ${ }^{47}$

\section{Passive targeting}

Nano-drug delivery systems (DDSs) have the capacity for passive targeting to tumor sites by capitalization of their size on the EPR phenomenon exhibited by tumor tissue. ${ }^{17,33}$ Characteristic of tumor tissue is a phenomenon termed the
EPR effect (illustrated in Figure 3). Of particular significance are the structural differences in the microvasculature of tumor tissue relative to that of vessels found in normal tissue. Tumors grow at an accelerated pace, rendering their demand for nutrients supplied via the vascular system greater than that of normal, healthy cells. In an effort to satisfy this amplified demand, rapid and hypervascularization occurs within tumor tissue. ${ }^{26,48,49}$ However, this microvasculature lacks the structural organization of normal blood vessels, exhibiting irregular diameters and branching patterns that compromise the integrity of the vessels. ${ }^{16,48,50}$ The endothelial cells found in the blood vessels of normal tissue may have gaps between them that are approximately 7-10 nm in size and are thus referred to as tight junctions. In tumor microvasculature, however, these gaps can range up to a few hundred nanometers, allowing the preferential extravasation of nanosystems in tumor tissue. ${ }^{26,33,48,49}$ Moreover, recovery of macromolecules via the vascular and lymphatic systems is compromised in tumor tissue, effectively resulting in retention of these macromolecules within tumor tissue. ${ }^{26,48,49}$ Passive targeting is thus a very efficient means of targeting with regard to tumor site-specific drug delivery, and nanoDDSs are able to capitalize on this phenomenon.

\section{Active targeting}

As previously mentioned, nanosystems are particularly amenable to the attachment of targeting moieties. The targeting nature of nanosystems can be tailored for specific targets and functions by the inclusion of targeting moieties such

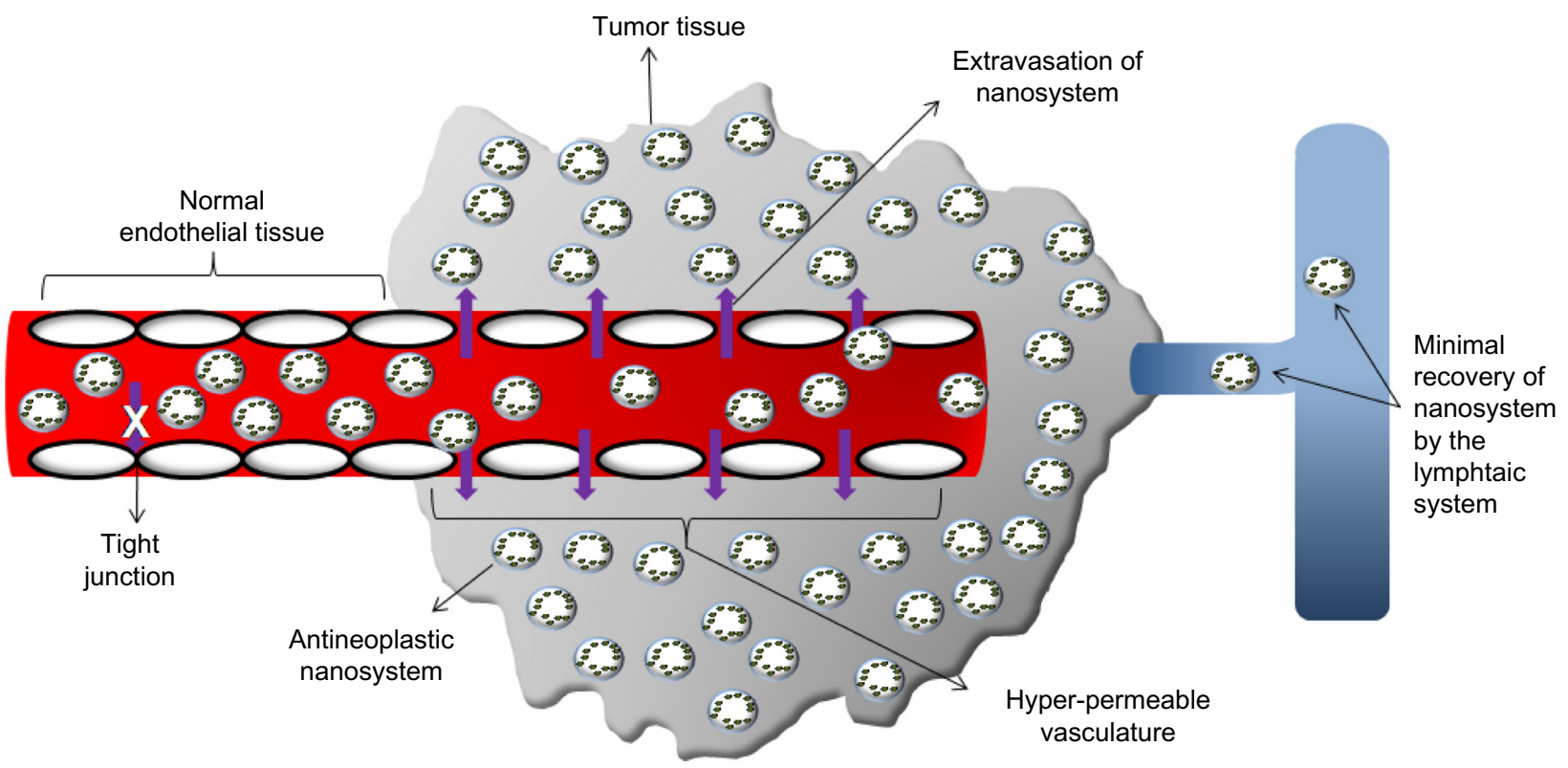

Figure 3 Diagrammatic representation of the factors contributing to the enhanced permeability and retention phenomenon displayed by tumor tissue. 
as peptides, antibodies, and carbohydrates. ${ }^{31-33,46,50}$ These functionalized nanosystems are thereby able to capitalize on the presence of receptors that are overexpressed, or expressed specifically, on the surface of the specific cancer subtype that is being targeted, by the formation of a ligandreceptor complex. Ideally, the target for antineoplastic drugs should be essential to the survival of tumor cells, as well as specific to tumor cells. ${ }^{47,52}$ However, targets that fulfill these characteristics are scarce and immensely difficult to isolate. The formulation constituents of the nanosystem, such as polymers, can also add to the targeting nature by responding to microenvironmental conditions or other physiological stimuli. These are termed "smart" or stimuli-responsive polymers and are able to modulate drug delivery in response to physiological changes that characterize the condition, such as the lower $\mathrm{pH}(\sim 6.5)$ of tumor tissue. ${ }^{52,53}$ Substantial research has been concentrated on isolating receptor targets specific to tumors, in general and for specific tumor subtypes, which can be used for drug delivery and imaging agents. These receptors with the prospective nanotechnological approach toward their targeting are discussed further.

\section{Folate receptor- $\alpha$}

Folate is a low-molecular weight vitamin essential to cellular function through its role in mitochondrial protein synthesis, amino acid metabolism, and the synthesis of nucleic acid precursors. ${ }^{54}$ Animals are unable to synthesize this micronutrient, and hence, the absorption of folate from exogenous sources is essential to satisfactory cellular function. Folate receptors, ubiquitously present throughout the body, facilitate cellular uptake of folate by endocytosis. Folate receptor- $\alpha$ is a glycosylphosphatidylinositol-anchored protein and one of the most prevalent receptors investigated for targeting, despite its ubiquitous presence in the body, on account of its significant overproduction and presence on the surface of a wide spectrum of tumors, particularly those of epithelial origin. ${ }^{24,26,55,56}$ Folic acid, the ligand responsible for binding to this receptor, is a popular ligand, as it is small and nonimmunogenic, has a high affinity for the folic acid receptor, and is hardly problematic to procure. ${ }^{29}$ Table 1 briefly outlines different nanosystem configurations that have been functionalized for targeting the folate receptor.

\section{Heat shock protein 90 (Hsp90)}

Hsp90, an intracellular molecule involved in signal activation, is widely present throughout the body, yet overexpressed in several cancers, such as ovarian cancer, head and neck cancers, esophageal cancers, and leukemia. ${ }^{61,62}$ This procar-
Table I Nanosystems functionalized for folate receptor targeting in cancer therapeutics

\begin{tabular}{|c|c|c|}
\hline $\begin{array}{l}\text { Type of } \\
\text { nanosystem }\end{array}$ & Brief description & Reference \\
\hline $\begin{array}{l}\text { Magnetic } \\
\text { nanoparticles }\end{array}$ & $\begin{array}{l}\text { Superparamagnetic nanoparticles } \\
\text { coated with temperature-sensitive } \\
\text { polymer and folic acid, for tumor } \\
\text { imaging and treatment. }\end{array}$ & 56 \\
\hline Quantum dots & $\begin{array}{l}\text { Stabilized core/shell zinc sulfide:Mn } \\
\text { sulfide quantum dots, functionalized } \\
\text { with folic acid for tumor imaging. }\end{array}$ & 24 \\
\hline Nanocrystals & $\begin{array}{l}\text { Rare-earth oxide nanocrystals } \\
\text { functionalized with folic acid, for } \\
\text { bimodal tumor imaging. }\end{array}$ & 28 \\
\hline $\begin{array}{l}\text { Thermosensitive } \\
\text { magnetic } \\
\text { liposomes }\end{array}$ & $\begin{array}{l}\text { Folate-targeted liposomes that } \\
\text { respond to induced localized } \\
\text { hyperthermia, for biological and } \\
\text { physical tumor targeting. }\end{array}$ & 57 \\
\hline $\begin{array}{l}\text { Supramolecular } \\
\text { vesicle aggregates }\end{array}$ & $\begin{array}{l}\text { A self-assembling polymeric system } \\
\text { containing gemcitabine, conjugated } \\
\text { to folic acid for targeted cancer } \\
\text { therapy. In vivo studies highlighted } \\
\text { superior activity of the targeted } \\
\text { system relative to a nontargeted } \\
\text { system. }\end{array}$ & 58 \\
\hline $\begin{array}{l}\text { Targeted } \\
\text { nanoparticle }\end{array}$ & $\begin{array}{l}\text { A nanoparticle consisting of a } \\
\text { heparin-folate-paclitaxel conjugate } \\
\text { with paclitaxel in the core of the } \\
\text { nanoparticle. This system was } \\
\text { designed to minimize P-glycoprotein- } \\
\text { mediated tumor resistance to } \\
\text { therapy and demonstrated promising } \\
\text { results in the treatment of head and } \\
\text { neck cancers in an animal model. }\end{array}$ & 59,60 \\
\hline
\end{tabular}

Abbreviation: $\mathrm{Mn}$, manganese.

cinogenic molecule is thus an attractive target as a treatment modality for cancer. 17-allylamino-17-demethoxy geldanamycin (17-AAG) has been identified as an inhibitor of Hsp90 because of binding to the adenosine triphosphate (ATP)binding site on the N-terminus of Hsp90, thereby blocking the activity of this compound and, in turn, preventing tumor cell proliferation. ${ }^{61}$ Yet again, poor aqueous solubility and hepatotoxicity render this compound clinically of no use. The favorable characteristics that define nanosystems, however, augur well for the future use of this compound in cancer therapy. Onyüksel et al successfully formulated 17-AAG in long-circulating, sterically stabilized phospholipid nanomicelles (SSMs). ${ }^{61}$ These SSMs were further functionalized with vasoactive intestinal peptide, a receptor that is overexpressed on the tumor membrane of many cancers. ${ }^{61}$ First, the successful solubilization of therapeutically significant concentrations of 17-AAG was achieved with the SSM. Second, the functionalized SSMs displayed superior toxicity as a result of increased cellular uptake. ${ }^{61}$ Unexpectedly, nanotargeted 
systems displayed suboptimal cytotoxic properties in the testing period compared with free 17-AAG. However, this may be a result of delayed release of SSM-associated 17-AAG. It must be borne in mind, however, that ex vivo cytotoxicity was not the challenge with 17-AAG; rather, stability and toxicity challenges needed to be overcome. The nanosystem addressed these shortcomings, whereas the functionalized system retained the cytotoxic capacity. The size range achieved $(\sim 16 \mathrm{~nm})$ allows this DDS to escape clearance by the reticuloendothelial system, whereas the properties of poly(ethylene glycol) (PEG) contribute to the long circulation lifespan, which cumulatively allow a greater chance for interaction with, and internalization into, target cells. ${ }^{61}$ In addition to the ATP-binding site mentioned earlier, a second ATP-binding site is present on the $\mathrm{C}$ terminus. This site has displayed affinity for other antineoplastic agents such as cisplatin, novobiocin, and epigallocatechin-3-gallate. ${ }^{62}$

\section{Human epidermal growth factor receptor 2 receptor}

Human epidermal growth factor receptor 2 (HER-2) has emerged as a prominent receptor target overexpressed in breast and ovarian cancer. Breast cancer is one of the most prevalent tumor types among women, with 20\%-30\% presenting with HER-2-positive tumors that demonstrate chemoresistance to some antineoplastic agents, and an overall poor prognosis. ${ }^{63}$ Trastuzumab $^{\circledR}$ is a humanized monoclonal antibody developed by Genentech (San Francisco, CA, USA) in the late 1980s, to target HER-2 and has been successfully used clinically in the treatment of HER-2-positive breast cancer. ${ }^{63,64}$ Lei et al highlighted the increased uptake of functionalized nanoparticles containing doxorubicin (DOX) by ovarian cancer cell lines. ${ }^{33}$ These nanoparticles were conjugated with an anti-HER-2 ligand to form a complex with the HER-2 receptor that displays preferential expression on the surface of ovarian cancer cells. The functionalized nanoparticles exhibited increased uptake by the cancer cells, because of a receptor-mediated endocytosis, compared with their nonfunctionalized counterparts. ${ }^{33}$ Several other receptors have also been identified as potential targets in cancer chemotherapy. Epithelial cell adhesion molecule is a transmembranous glycoprotein that is overexpressed on the surface of solid tumors, but not on normal tissue. ${ }^{65}$ On binding, the receptor-ligand complex is rapidly internalized, which is particularly advantageous for the delivery of drugs with intracellular targets. ${ }^{65}$ Vasoactive intestinal peptide is overexpressed on the tumor membrane of many cancers and is therefore a possible target. ${ }^{61}$ Other potential targets that have been identified including tumor associated glycoprotein 72
(TAG-72), luteinizing hormone-releasing hormone receptor, follicle stimulating hormone receptor, transferrin receptor, and heparin-binding glycoproteins such as VEGF. 25,31,32,66

\section{Aptamer-based targeted nanotherapy}

Aptamers are a class of oligonucleotides comprising synthetic single-stranded DNA or RNA molecules that can be incorporated into DDSs for highly specific targeting to various molecular targets present on tumor cells. ${ }^{67}$ Aptamers may be conjugated to drug molecules or DDSs by intercalation of covalent bonding. Various nanoconstructs have been assessed for aptamer functionalization, such as nanorods, nanoparticles, and carbon nanotubes, to improve targeting and uptake of the DDSs by tumor cells. AS1411 was the first DNA aptamer to enter clinical oncology trials. ${ }^{68}$ This aptamer has demonstrated extraordinary specificity as well as binding affinity for its plasma membrane target receptor, nucleolin, which is overexpressed in tumor cells. Yang et al formulated a gold nanorod DDS with a mesoporous, AS1411 surfacefunctionalized silica framework. ${ }^{69}$ This photothermal DDS is activated by illumination in the near-infrared region, resulting in structural changes and consequent drug release. In vitro results highlighted versatility of the DDS, as well as biocompatibility, high tumor specificity, and appropriate drug release for intracellular targets. ${ }^{69}$ Functionalization of a liposomal DDS with the sgc8 aptamer, which is specifically targeted to protein tyrosine kinase present on CEM-CCRF leukemia cells, was undertaken by Kang et al. ${ }^{70}$ The high aptamer load resulted in increased binding affinity to target tumor cells with no compromise on formulation stability. In vitro studies confirmed the selectivity of the functionalized liposomal DDS for the target cell, as well as intracellular delivery of the drug to tumor cells. ${ }^{70}$ However, this system did not include in vivo studies, which are essential to the critical assessment of the targeting efficacy of DDSs.

\section{CD44 receptor}

CD44 is a membrane glycoprotein present on the surface of various solid tumors, particularly non-small cell lung cancer, for which it is considered a biomarker. ${ }^{71} \mathrm{CD} 44$ is the primary receptor for hyaluronic acid (HA), through which HA acts to regulate cell proliferation and passage. ${ }^{71}$ Platt and Szoka have extensively reviewed the interaction of HA and CD44 and the potential of the receptor, substrate, or complex as targets in tumor therapy. ${ }^{71}$ The incorporation of HA into nanosystems has been widely investigated for targeting to tumor sites. Ganesh et al formulated HA-based self-assembling nanoconstructs with a small interfering RNA therapeutic 
payload. ${ }^{72}$ In vitro and in vivo studies undertaken in this study highlight high cellular internalization in cells expressing CD44, whereas the mechanism of cellular internalization was confirmed to be CD44-mediated endocytosis. ${ }^{72}$ This mechanism of cellular internalization in CD44-expressing cells was further confirmed by Gary-Bobo et al. ${ }^{73}$ This team reported on a mesoporous silica nanoparticle coated with poly-(L-lysine) and HA for photodynamic therapy (PDT). Cellular uptake and phototoxicity studies were undertaken on an HCT116 breast cancer cell line. HA-functionalized nanoparticles demonstrated significantly higher phototoxicity relative to nonfunctionalized nanoparticles. ${ }^{72}$

\section{Tackling emergence of drug resistance in tumors through nanotechnology}

Multidrug resistance (MDR) has emerged as an acute problem contributing to the already diverse and significant challenges compromising the success of cancer chemotherapy. ${ }^{55,74}$ MDR can manifest as a failure of initial treatment protocols to adequately reduce tumor size or, as is the case most often with ovarian cancer, as a relapse/ recurrence after successful therapy. ${ }^{31,55}$ The high rate of recurrence in ovarian cancer is the primary contributing factor to the poor prognosis associated with ovarian cancer and the low 5-year survival rate. MDR currently accounts for treatment failure in more than $90 \%$ of patients presenting with metastasis, which in the case of ovarian cancer is the majority of patients. ${ }^{75}$ Drug resistance displayed by tumors can be classified as decreased drug influx into tumor cells, increased drug efflux out of tumor cells, activation of DNA repair, metabolic deactivation of antineoplastic drugs, and simultaneous inactivation of apoptotic pathways and activation of protective mechanisms of cellular antiapoptotic pathways. $^{75}$

The application of nanotechnology has shown immense potential in overcoming or circumventing the extra- and intracellular mechanisms associated with drug resistance. The most significant application has been to overcome the resistance to DOX, which has displayed substantial success and will be discussed later. Among the extracellular resistance mechanisms that nanosystems will find applicability in is the inactivation of drug molecules before their reaching the site of action. Several nanosystems offer protection from unsuitable environments and metabolic and enzymatic degradation, as well as protection against early clearance from the body. Coating with compounds such as PEG allows nanosystems to navigate the body undetected by clearance systems, thereby increasing the circulation half-life. ${ }^{55}$
The internalization of free drug into tumor cells is primarily through passive diffusion, whereas the mechanism of nanosystem entry into tumor cells involves nonspecific endocytosis. ${ }^{75}$ A primary mechanism of resistance in cancer cells is increased drug efflux out of cells by efflux pumps. The passive diffusion of free drug allows for recognition by and activation of efflux pumps, reducing cellular drug concentration and compromising antineoplastic therapy. ${ }^{75}$ However, the mechanism of nanosystem internalization facilitates undetected entry into cells, bypassing the increased drug efflux mechanism of drug resistance in tumor cells, as illustrated in Figure $4 .^{75}$

\section{Antineoplastic drug challenges: nanotechnology solutions DOX}

DOX, an anthracycline antibiotic, is one of the most efficacious drugs in the treatment of several solid tumors, including breast and ovarian cancers. ${ }^{33,49,77}$ However, clinical application of DOX has been severely hindered because of its critical toxicity profile, the foremost of which being cardiotoxicity. ${ }^{49,77}$ DOX-loaded nanoparticulate formulations have been investigated as a possible means of reducing detrimental adverse effects, as well as increasing the efficacy of DOX, to preserve the use of the potent, widely applicable antineoplastic agent. ${ }^{77}$ Several materials have been examined for their potential in this application, with DOX being incorporated into or bound to the nanoparticles. Among these materials are poly(butyl cyanoacrylate), poly(lactic-co-glycolic acid) (PLGA), poly(isohexal cyanoacrylate), and gelatin. ${ }^{77,78}$ Nanoparticulate formulations for DOX have been immensely successful, with formulations displaying passive targeting on account of the nanoscale sizing and the ability to cross the blood-brain barrier for the treatment of brain tumors, as well as success against several lines of drug-resistant cancer cells. ${ }^{77}$ Human serum albumin has also been explored as a nanoparticulate carrier for DOX, with appreciable success. ${ }^{77}$ At this time, there are two commercially available registered human serum albumin-based formulations (namely, Albunex and Abraxane), which drove research into the viability of this material as a nanocarrier. ${ }^{77}$

The development of MDR in cancer cells has further compromised the feasibility of DOX use. ${ }^{33,55}$ MDR is a result of the overexpression of the transmembranous protein, P-glycoprotein (P-gp), which compromises the therapeutic efficacy of DOX. ${ }^{33,65}$ Increasing the dose of DOX to overcome this drawback is not viable because of the abovementioned dose-related adverse effects. ${ }^{33}$ Lei et al developed 


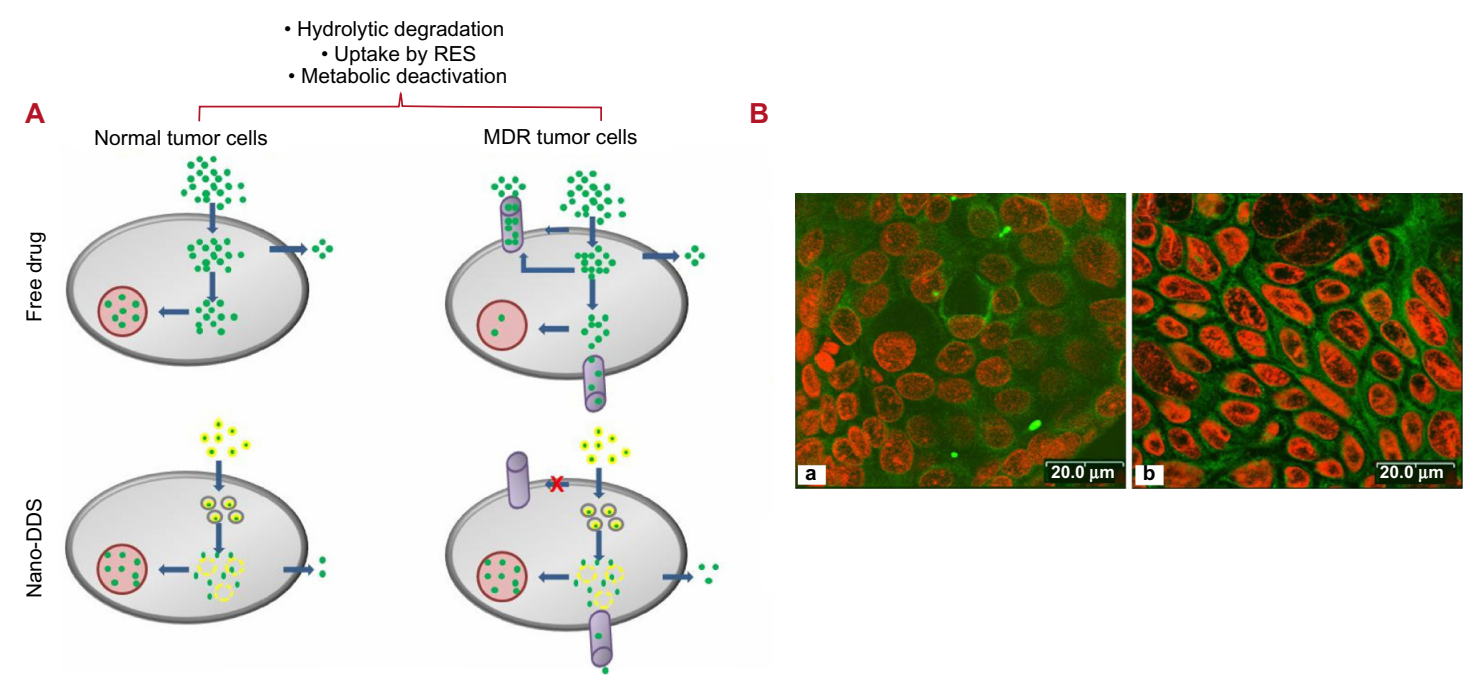

Figure 4 (A) Diagrammatic comparison of the internalization mechanisms of free drug and nanodrug delivery systems in normal tumor cells and multidrug-resistant tumor cells. ${ }^{75}$ (B) Confocal microscopic images of Caco-2 cells after I hour incubation at $37{ }^{\circ} \mathrm{C}$ with coumarin 6-loaded PLGA nanoparticles coated with (a) PVA or (b) vitamin E TPGS. The cells were stained by propidium iodide (red) and uptake of green fluorescent 6 -coumarin-loaded nanoparticles in Caco- 2 cells was visualized by overlaying images obtained by FITC filter and RITC filter. The two figures show a distinct extent in cellular uptake of the nanoparticles. ${ }^{76}$

Note: (A) Reprinted from Journal of Controlled Release, 162(I), Gao Z, Zhang L, Sun Y, Nanotechnology applied to overcome tumor drug resistance, 45-55, Copyright @ 20I2, with permission from Elsevier. (B) Reprinted from Biomaterials, 26(I5), Win KY, Feng S-S, Effect of particle size and surface coating on cellular uptake of polymeric nanoparticles for oral delivery of anticancer drugs, 27I3-2722, Copyright (C) 2005, with permission from Elsevier.

Abbreviations: DDS, drug delivery system; MDR, multidrug resistance; RES, reticuloendothelial system; PLGA, poly(lactic-co-glycolic acid); PVA, polyvinyl alcohol; vitamin E TPGS, vitamin E succinated polyethylene glycol 1000; FITC, fluorescein isothiocyanate; RITC, rhodamine B isothiocyanate.

DOX-encapsulated PLGA nanoparticles and observed a significant increase in cellular uptake of the nanoparticulate DDS compared with free DOX, which was attributed to a difference in the mechanism of cellular uptake. ${ }^{33}$ The free form of DOX passes into the cell via diffusion, whereas the nanoenabled DDS was taken up via endocytosis, which proved more favorable in cells displaying an overexpression of P-gp. ${ }^{33}$

The capacity of targeted nanoenabled DDSs to accumulate in target tissue has further proved beneficial with regard to the administration of DOX in P-gp-negative cells. Drug resistance can be a consequence of alteration of the intracellular $\mathrm{pH}$-gradient, which results in drugs being trapped in cellular components, where they cannot exert their action. ${ }^{33}$ Cytotoxic studies undertaken by Lei et al highlighted the increased cytotoxic effect of functionalized and nonfunctionalized DOX-containing PLGA nanoparticles compared with free DOX at higher concentrations. ${ }^{33}$ The research team rationalized that the DOX-containing nanoparticles were entrapped in lysosomes or endosomes within the cell and that the release of DOX into the cytosol was limited by an unfavorable $\mathrm{pH}$ gradient. The DOX was therefore unable to reach the site of action, the cell nucleus. This resulted in limited cytotoxicity at low concentrations. However, at elevated concentrations, the concentration of DOX from the nanoparticles surpasses the cells' lysosomal and endosomal capacity, resulting in increased DOX in the cytosol of the cell, with uninhibited movement to the cell nucleus, where it can exert its effect. ${ }^{33}$
Poly(alkyl cyanoacrylate) nanoparticles have also shown tremendous potential in overcoming MDR to DOX. However, the mechanism to overcome drug resistance appears to differ from that of PLGA nanoparticles, in that poly(alkyl cyanoacrylate) nanoparticles are not endocytosed into cells but, rather, adhere to the surface of tumor cells. ${ }^{55}$ The drug is subsequently released and combines with the degradation products of the nanoparticles, forming an ion pair that diffuses into the tumor cell undetected by P-gp.

Yet another attempt at overcoming tumor cell resistance to DOX was the coencapsulation of DOX and the chemosensitizing agent cyclosporin A within a single nanoparticle. ${ }^{55}$ DOX formed the core of the nanoparticle, covered by the cyclosporin A. This configuration allowed for the rapid release of cyclosporin A and resulting sensitization, followed by the release of DOX. Formulation as a nanoparticle ensured both agents reached the desired site of action via passive targeting, thereby potentiating the activity of the individual agents and their synergistic activity, while reducing harmful effects on healthy tissue..$^{55}$

\section{Camptothecin}

Camptothecin (CPT) was first isolated in 1966 from the tree Camptotheca acuminata ${ }^{79,80} \mathrm{CPT}$ is a potent antineoplastic drug that has activity against a vast array of solid tumors, including ovarian, gastric, breast, cervical, melanoma, skin, and lung cancers. ${ }^{80-82} \mathrm{CPT}$ inhibits and stabilizes the 
topoisomerase I enzyme, which is required for cell replication and transcription and is overexpressed in certain cancer cells, but not in normal tissue, thereby inducing apoptosis of the cancer cells. ${ }^{79,81,83,84}$ However, use of this drug in clinical practice has been diminished because of its poor aqueous solubility, insolubility in most biocompatible organic solvents, instability under physiological conditions, and severe toxicity profile. ${ }^{81,82,84,85}$ The chemical composition of CPT contains an active lactone group, which is responsible for its insolubility and physiologically labile properties, yet crucial to its antineoplastic activity. ${ }^{79,81,83,86}$ Figure 5 illustrates the reversible conversion from lactone to carboxylate form under physiological conditions. It is therefore essential to protect this lactone group in the formulation and delivery of CPT preparations. Although derivatives of CPT are used, they lack the potency of the parent drug. ${ }^{82,86}$ Furthermore, CPT and its derivatives usually require continuous infusion over a period of time, or multiple injections over a short period, which is a great disadvantage with regard to patient comfort and acceptability. ${ }^{87}$ In addition, prolonged administration results in intolerable adverse effects, including neutropenia, anemia, and other related hematologic manifestations, altered gastric function, and dermatological manifestations. ${ }^{87}$

Several methods have been investigated to improve the solubility profile of CPT. ${ }^{84}$ Although formulation of CPT as liposomes, microemulsions, and polymeric microspheres has enjoyed a degree of success, incorporation into a nanoDDS may hold greater potential for the clinical use of this drug. ${ }^{83,84,87}$ An emphasis has also been placed on the development of controlled release DDSs, as it is hypothesized that the properties of these systems will be able to adequately address the factors limiting CPT use clinically. ${ }^{87}$
Micelles are composed of phospholipids that take on a spherical form spontaneously in aqueous conditions, above the critical micellar concentration. ${ }^{81}$ These moieties consist of a hydrophobic core and hydrophilic corona, thereby enabling the solubilization of poorly aqueous soluble drugs and their delivery in an aqueous medium, the likes of which will be required for intravenous delivery. ${ }^{38,80}$ In addition, the nanodimensions of micelles allow for their easy passage through the more permeable vasculature that predominates in cancer tissue, making them valuable in cancer therapy. ${ }^{80}$ The ease of manufacture, reproducibility, and stability of micelles make them attractive systems for drug delivery.

Aiming to investigate methods of safely and effectively delivering CPT to the intended site of action (ie, tumor tissue), Koo et $a^{81}$ developed SSMs. Distearoylphosphatidylethanolamine conjugated to PEG-2000 was employed as the lipid phase in the current study because of its biocompatibility and biodegradability, as well as the potential to increase the circulation half-life of the system by steric stabilization. Koo et al demonstrated an approximately 25 -fold increase in the solubility of CPT in the optimized formulation compared with free CPT. ${ }^{81}$ Moreover, the researchers reported an increased aqueous stability with preservation of the active lactone moiety as a result of drug encapsulation within the hydrophobic core of the SSM, rather than being exposed to the aqueous environment, where hydrolytic degradation can occur. ${ }^{81}$ Because CPT acts on cells in the S-phase of the cell cycle, formulations with a longer half-life will have a greater cytotoxic effect. The SSM formulation, because of its increased stability and circulation half-life, displayed threefold greater cytotoxicity than free CPT after 24 hours, when tested against breast cancer cells, because of a greater proportion of tumor cells being exposed to CPT. The

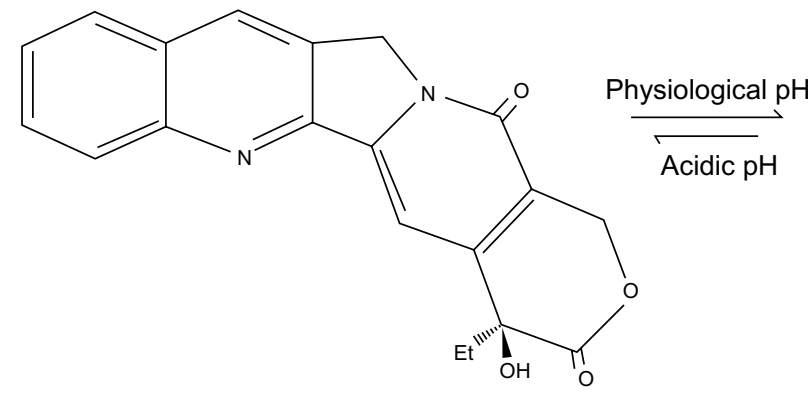

- Poor aqueous solubility

- Extremely potent antineoplastic activity

- Low affinity for HSA

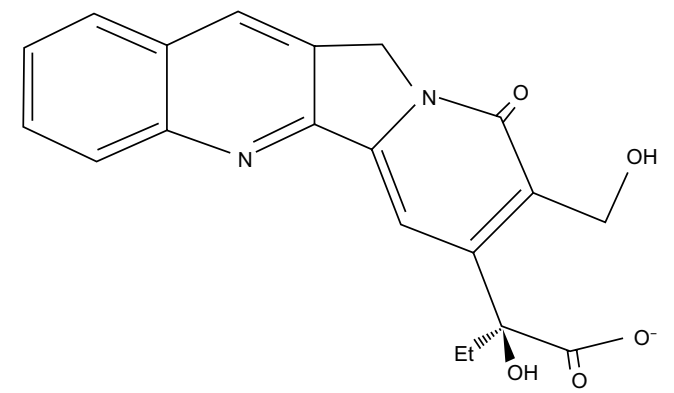

- Increased aqueous solubility

- Severely diminished antineoplastic activity

- High affinity for HSA

Figure 5 Illustration of the change in chemical structure from the highly active lactone form to the less active carboxylate form of camptothecin at physiological pH. Abbreviation: HSA, human serum albumin. 
augmented cytotoxicity may also be attributable to increased cellular uptake and accumulation of the nanosystem in tumor tissue. ${ }^{81}$ The aforementioned findings were supported by results obtained by $\mathrm{Mu}$ et al, who produced mixed micelles as CPT delivery systems. ${ }^{80}$ In addition to demonstrating ease of preparation and favorable pharmacokinetic properties, Koo et al highlighted the potential of this DDS for practical application in a clinical setting. ${ }^{81}$ The SSM developed displayed negligible alterations in size, solubility, and the wavelength of peak fluorescence emission after lyophilization and reconstitution. ${ }^{81}$ This has significant implications for storage and use in a clinical setting. The incorporation of PEG was attributed to the stability to freeze drying, as PEG has cryoprotectant and lyoprotectant properties. ${ }^{81}$ The CPT-loaded SSMs prepared in this study displayed immense potential for application in clinical practice because of their superior efficacy and practicality of use.

To address the above-mentioned shortfalls of CPT, Fang et al formulated a CPT-containing nanoemulsion DDS. ${ }^{82}$ Although the primary focus of this study was investigation of physicochemical properties of the prepared nanoemulsions, their in vitro and ex vivo studies delineated promising results. Varying cytotoxic profiles were obtained for the nanoemulsions prepared, as a function of changes in formulatory composition. In addition, the study did not take into account the effect of metabolism, enzymatic degradation, and other processes, which the system will undergo in the body and that will greatly affect the nature of the DDS that ultimately reaches the target site. However, the superior drug-loading capacity (90\%) and low hemolytic destruction highlights the potential of a nanoenabled DDS for a very useful, potent drug.

CPT-containing nanoparticles have been vigorously investigated in the quest to develop an efficacious DDS. ${ }^{84,88}$ This nanosystem flaunts prolonged vascular circulation, particularly when coated with PEG, allowing for greater interaction between tumor tissue and antineoplastic drug, as well as superior cellular uptake of CPT, resulting in tumor cells being bombarded, and thereby having enhanced cytotoxic effect. ${ }^{84}$ However, CPT loading capacity of nanoparticles remains the limiting factor in successful formulation.

\section{Paclitaxel}

Paclitaxel (PTX) is a microtubule-stabilizing agent that acts to disrupt cell division, causing cell death. ${ }^{49,89}$ It is currently considered first-line treatment for ovarian cancer. PTX is a strongly hydrophobic compound, which poses significant formulation problems for intravenous administration. ${ }^{32,90}$ Solubilization in Kolliphor ${ }^{\circledR}$ EL (SigmaAldrich, St Louis, MO, USA) has been successful, with the only currently available PTX formulation being the intravenously administered Taxol ${ }^{\circledR}$ (Bristol-Myers Squibb, New York, NY, USA). ${ }^{90}$ However, this compound causes significant undesirable adverse effects, making the development of delivery systems that do not require Kolliphor ${ }^{\circledR}$ EL appealing. ${ }^{89}$

Nanoparticles have been the most widely explored nanosystem for the delivery of PTX, with a variety of materials and functionalization techniques having been explored. ${ }^{32}$ PTX nanoparticles have been widely successful in addressing the problems associated with PTX administration and efficacy; that is, the nanoparticles have successfully solubilized PTX and displayed adequate drug loading capacity; drug delivery is more targeted, even in nonfunctionalized nanoparticles, because of passive diffusion; and increased therapeutic efficacy in vivo, as a result of targeted delivery, increased cellular uptake, protection of PTX from enzymatic degradation and inactivation, and an increase in circulation half-life. ${ }^{32}$

The incorporation of PTX into nanoparticles has been associated with significantly favorable outcomes, particularly with regard to the biodistribution and pharmacokinetic profile, relative to that of free PTX and Taxol ${ }^{\circledR} .{ }^{90}$ PLGA nanoparticles have been successfully prepared in an appropriate size range (117-160 $\mathrm{nm}$ ) for extravasation into tumor tissue, with extremely high loading efficiencies. ${ }^{49,90}$ Furthermore, when tested against human small-cell lung cancer cell lines, this nanosystem displayed an approximately $70 \%$ loss of cell viability. ${ }^{90}$ The ability of nanoparticles to overcome resistance mediated by P-gp is particularly beneficial to its application in PTX delivery, as there is already evidence of acquired resistance to this drug. ${ }^{90}$

\section{Nutlin-3a}

Nutlin-3a, a promising antineoplastic drug currently under investigation, acts to inhibit the inactivation of the p53 tumorsuppressing protein. ${ }^{65,91}$ A significant fraction of tumors display altered or inactivated p53 genes, which results in loss of the tumor-suppressing function. ${ }^{65}$ Inactivation of the p53 protein is also via attachment by human double minute 2 oncoprotein, which causes inactivation of p53. Nutlin3 a shares a binding site with p53 on the human double minute 2 oncoprotein, thereby preventing the attachment and inactivation of $\mathrm{p} 53 .{ }^{65}$ The potential of this drug in restoring the tumor-suppressing capacity of $\mathrm{p} 53$, and hence in treating cancer, is vast. 
However, there are many shortfalls limiting the use of this drug, such as poor solubility, nonspecific targeting, systemic toxicity, low bioavailability in tumor tissue, efflux by transmembranous proteins, and degradation by cellular lysosomes, resulting in decreased cellular concentration. ${ }^{65}$ Nanotechnology displays the propensity to overcome the above-mentioned shortfalls, allowing for the optimal use of this potentially valuable drug. Das and Sahoo formulated nonfunctionalized Nutlin-3a-containing PLGA nanoparticles (Nutlin-PLGANP) and epithelial cell adhesion moleculefunctionalized Nutlin-PLGANP for the treatment of colon and lung cancer. ${ }^{65}$ The effectiveness of nanosystems is a function of the drug loading efficiency, target cell uptake, and release of drug in the target cell. The functionalized and nonfunctionalized nanoparticles prepared by Das and Sahoo exhibited a high loading efficiency of Nutlin-3a, in the order of $\sim 78 \%$ and $\sim 81 \%$, respectively, along with a significantly increased cellular uptake compared with free Nutlin-3a. This team reported a fivefold and 16-fold increase in cellular uptake of nanoparticulate Nutlin-3a and the functionalized counterparts, respectively, compared with the free form. The investigators demonstrated augmented cytotoxicity by the unconjugated nanoparticles and a further increase by the conjugated nanoparticles compared with native Nutlin-3a. ${ }^{65}$ Although non-drug-loaded nanoparticles were not used as a control in this study to ascertain the effect of the system itself on cytotoxicity, other studies have demonstrated noncytotoxic activity by PLGA nanoparticles..$^{90,92}$ This work requires further in vivo analysis; however, the presented in vitro results are indeed promising.

\section{Indisulam}

Indisulam is a sulfonamide drug that interrupts the cell cycle at the $\mathrm{G}_{1} / \mathrm{S}$ phase. ${ }^{93}$ The cytotoxic effects have been established, particularly against breast cancer. However, on reaching clinical trials, dose-related toxicities unfavorably tipped the risk:benefit scale, rendering the drug unsuitable for clinical use. ${ }^{93}$ Aqueous indisulam formulations extravasate freely from the vascular system, resulting in toxicity to healthy tissue. Furthermore, they bind to erythrocytes and plasma proteins, causing hemotoxicity. ${ }^{93}$ Thus, indisulam requires a delivery vehicle to prevent interaction with plasma proteins and erythrocytes, as well as, ideally, to promote preferential accumulation in tumor tissue.

To this end, Cesur et al formulated a nanomicellar DDS incorporating indisulam for targeted delivery to breast cancer tissue. ${ }^{93}$ This simple, cost-effective method of preparation resulted in uniform, reproducible, and stable nanomicelles $\sim 15 \mathrm{~nm}$ in size. ${ }^{93}$ The size range achieved facilitates preferential accumulation of the DDS in tumor tissue as a result of extravasation from leaky vasculature. Solubility of indisulam in aqueous medium was substantially increased (about eightfold), allowing for the intravenous delivery of a greater concentration of the drug. Cytotoxicity against the chosen breast cancer cell line was augmented in the nanomicellar DDS compared with indisulam in dimethyl sulfoxide. In addition, the prepared formulation could be lyophilized to facilitate long-term storage without significant alteration of the properties. ${ }^{93}$ In spite of these promising results, this DDS will have to be subjected to in vivo evaluation and clinical trials before its full potential and safety can be ascertained.

\section{Curcumin}

An emerging alternative in antineoplastic therapy is the use of phytochemicals, either as a therapeutic modality on their own or in conjunction with commercially available antineoplastic drugs as a means of sensitizing tumor cells to conventional treatment. ${ }^{94}$ Curcumin, a hydrophobic polyphenol derived from the Curcuma longa herb, is one such entity that has displayed antineoplastic activity, among its wide range of therapeutic applications, as a result of interaction with the cell cycle process, angiogenesis, apoptosis, and metastasis. ${ }^{16,94-96}$ Furthermore, the clinically established safety profile of this phytochemical favor its use in cancer chemotherapy, with large doses proving tolerable. ${ }^{94}$ However, several pharmacokinetic challenges plague the successful clinical use of curcumin. Poor aqueous solubility poses formulatory and administration challenges, and degradation, poor tissue penetration and absorption, and rapid systemic elimination culminate in severely reduced bioavailability. $27,94-96$

Because the obvious potential of this compound cannot be disregarded, particularly in the face of growing exigency for successful antineoplastic treatment, efforts to overcome the above-mentioned challenges to curcumin use have produced nanosystems incorporating curcumin. ${ }^{94-97}$ PLGA nanoparticulate DDSs have shown variable, yet significant, increases in curcumin bioavailability, whereas other approaches have included incorporation in a phospholipid complex, as well as bioadhesive DDS. ${ }^{94}$ A particularly outstanding study conducted by Mohanty and Sahoo formulated highly stable glycerol monooleate-based curcumin-loaded nanoparticles that displayed a highly favorable pharmacokinetic profile. ${ }^{94}$ These nanoparticles displayed approximately $90 \%$ curcumin entrapment, as well as biphasic release amounting to 
$\sim 46 \%$ in 24 hours and $\sim 66 \%$ over the course of 10 days, the characteristic of which would be favorable in cancer therapy. The nanoparticulate system exhibited greater aqueous solubility and stability than native curcumin, indicating protection from hydrolytic degradation conferred on curcumin by the nanosystem. ${ }^{94}$ Nanoparticulate DDS for curcumin showed augmented cellular uptake compared with free curcumin (5.9-7.7 times greater) and a substantially greater antiproliferative effect on tested cancer cell lines, as well as a greater (3.8 times) induction of apoptosis. Toxicity studies of nondrug-loaded nanoparticles proved the safety of this carrier system and the materials employed. The study was further extended to in vivo analysis, where higher levels of systemic curcumin were detected for longer periods of time after the administration of curcumin-loaded nanoparticles. ${ }^{94}$ These findings were far superior to those from previous studies on PLGA nanoparticulate curcumin, which was attributed to the controlled and prolonged manner of release from glycerol monooleate nanoparticles.

Anitha et al employed a straight-forward, easily upscalable method to formulate modified chitosan nanoparticles $(<200 \mathrm{~nm})$ incorporating curcumin to address the aforementioned shortfalls of curcumin bioavailability. ${ }^{96}$ The substantially favorable drug entrapment was comparable with that achieved by Mohanty and Sahoo $(87 \%$ versus $90 \%$ ), with controlled drug release achieved over the course of several days. Furthermore, cell viability studies indicated the retention of antitumor activity by curcumin despite loading into nanoparticles, and fluorescence spectroscopy confirmed the release of curcumin within cancer cells with resultant apoptosis. The results of the studies presented here have been corroborated by several other studies reporting on various nano-DDSs, which successfully addressed the challenges limiting the use of curcumin and highlighted the feasibility of nanoenabled curcumin as an antineoplastic agent. ${ }^{95,97-101}$

As the annals of nanomedicine research expand, it is the translation of nanosystems from benchtop to bedside that substantiates the clinical relevance of this field of research. Although currently small in number, the nanosystems that have received approval by the US Food and Drug Administration (FDA) have demonstrated considerable clinical success and are now firmly embedded in the treatment protocol of specific tumor subtypes. Moreover, the significant number of nanosystems undergoing preclinical investigations inspires an expectation of the imminent approval and clinical availability of more innovative nanosystems for cancer treatment. Table 2 provides a brief overview of commercially available as well as preclinical nanosystems for cancer therapy.

\section{Contemporary nanotechnological cancer therapies}

\section{Nanoenabled antisense oligonucleotides in cancer therapeutics}

Antisense oligonucleotides are fragments of deoxynucleotide that are complementary in sequence to a defective portion of messenger (m)RNA and act to interrupt the translation of defective proteins from such mRNA. ${ }^{114}$ Hence, antisense oligonucleotides have potential in cancer therapeutics by blocking the expression of defective genes. However, these moieties are labile and display poor cell penetration, necessitating a suitable carrier system for their successful clinical application. ${ }^{114,115}$ Formulation as nanoparticles and liposomes has been investigated to address these shortcomings of antisense oligonucleotides. ${ }^{115}$ Furthermore, the capacity of nanosystems for targeting aids in the use of antisense nucleotides, as it will prevent nonspecific binding.

Nafee et al formulated chitosan-coated PLGA nanoparticles and demonstrated the effect of formulation parameter variation in tailoring the properties of this nanosystem. ${ }^{116}$ Their study further demonstrated the successful encapsulation and intracellular delivery of the antisense oligonucleotide, 2'-O-methyl-RNA. ${ }^{116}$ A study undertaken by Zhong and team culminated in the formulation of midkinase antisense oligonucleotide (MK-ASODN) encapsulated into liposomes that are feasible for industry-scale production and that exhibited long-term stability. ${ }^{117}$ This nanosystem is intended for the treatment of hepatocellular carcinoma and demonstrated significant inhibition of three human hepatocellular carcinoma cell lines. ${ }^{117}$ In vivo investigations in a mouse model highlighted superior efficacy of the MK-ASODN liposomal system relative to nonencapsulated MK-ASODN. ${ }^{117}$

An innovative nanoresonator chip-based RNA sensor was developed by Sioss et al for the detection of circulating tumor cells. ${ }^{118}$ This study involved the covalent attachment of antisense oligonucleotides to nanowires, followed by the bottom-up assembly to create a nanoresonator. ${ }^{118} \mathrm{~A}$ high degree of sensitivity and selectivity of this RNA sensor for the PCA3 prostate cancer biomarker was established. The ability to control the integration of nanowire incorporation in biosensing devices to maximize exposure of detection moieties, as well as the ability to integrate varied antisense oligonucleotides, underlies the growing interest in this nanostructure for application in the detection and diagnosis of numerous tumor subtypes. 
Table 2 A brief outline of commercially available nanosystems and nanosystems undergoing preclinical investigations, in cancer therapeutics

\begin{tabular}{|c|c|c|c|c|c|}
\hline Product & $\begin{array}{l}\text { Type of } \\
\text { nanosystem }\end{array}$ & $\begin{array}{l}\text { Manufacturer and year } \\
\text { of US Food and Drug } \\
\text { Administration approval }\end{array}$ & Indication & Description & References \\
\hline \multicolumn{6}{|c|}{ Commercially available nanosystems } \\
\hline Doxil $^{\circledR}$ & $\begin{array}{l}\text { Liposome } \\
(\sim 100 \mathrm{~nm})\end{array}$ & $\begin{array}{l}\text { Alza Corporation } \\
\text { (1995) }\end{array}$ & $\begin{array}{l}\text { Refractory ovarian } \\
\text { carcinoma, Kaposi's } \\
\text { sarcoma }\end{array}$ & $\begin{array}{l}\text { First marketed product employing } \\
\text { stealth technology. Significant redu- } \\
\text { ction in cardiotoxicity associated } \\
\text { with doxorubicin. }\end{array}$ & 102,103 \\
\hline DaunoXome ${ }^{\circledR}$ & $\begin{array}{l}\text { Liposome } \\
(60-80 \mathrm{~nm})\end{array}$ & $\begin{array}{l}\text { NeXstar Pharmaceutical } \\
\text { (1996) }\end{array}$ & Kaposi's sarcoma & $\begin{array}{l}\text { Intravenous liposomal } \\
\text { formulation of anthracycline } \\
\text { daunorubicin exhibiting reduced } \\
\text { cardiotoxicity in vivo because of } \\
\text { a passive targeting mechanism. }\end{array}$ & 104 \\
\hline Emend $^{\circledR}$ & Nanocrystal & $\begin{array}{l}\text { Merck and Co., Inc. } \\
(2003)\end{array}$ & $\begin{array}{l}\text { Chemotherapy- } \\
\text { associated nausea }\end{array}$ & $\begin{array}{l}\text { Nanocrystals of the substance } \\
\text { P antagonist, aprepitant, } \\
\text { formulated as such to enhance } \\
\text { the water solubility and maximize } \\
\text { bioavailability from the narrow } \\
\text { absorption window of this drug. }\end{array}$ & 105 \\
\hline Abraxane $^{\mathrm{TM}}$ & $\begin{array}{l}\text { Albumin } \\
\text { nanoparticle } \\
(\sim 130 \mathrm{~nm})\end{array}$ & $\begin{array}{l}\text { American Pharmaceutical } \\
\text { Partners Inc and American } \\
\text { BioScience (2005) }\end{array}$ & $\begin{array}{l}\text { Refractory metastatic } \\
\text { breast cancer, non-small } \\
\text { cell lung cancer }\end{array}$ & $\begin{array}{l}\text { Injectable formulation containing } \\
\text { paclitaxel, employing innovative } \\
\text { nanoparticle albumin bound } \\
\text { technology to achieve tumor } \\
\text { targeting. }\end{array}$ & $106-108$ \\
\hline Product & Drug & Size, nm & Company & Description & References \\
\hline \multicolumn{6}{|c|}{ Nanosystems in late-stage preclinical trials } \\
\hline NK91। & Doxorubicin & 40 & Nippon Kayaku & $\begin{array}{l}\text { Polymeric micellar system } \\
\text { consisting of a block copolymer } \\
\text { of polyethylene glycol } \\
\text { poly(aspartic acid). }\end{array}$ & 109,110 \\
\hline Genexol $^{\circledR}$ & Paclitaxel & $<50$ & Samyang & $\begin{array}{l}\text { Micellar drug delivery system } \\
\text { formulated with the amphiphilic } \\
\text { polymer monomethoxy-poly } \\
\text { (ethylene glycol)-b-poly(D,L-lactide). } \\
\text { Response rate of metastatic breast } \\
\text { cancer to Genexol }{ }^{\circledR} \text { was superior } \\
\text { to Abraxane }{ }^{\circledR} \text { and Taxol }{ }^{\circledR} \text { in } \\
\text { Phase II and Phase III clinical trials. } \\
\text { Efficacy against non-small cell lung } \\
\text { cancer and pancreatic ductal } \\
\text { adenocarcinoma has also been } \\
\text { established in clinical trials. }\end{array}$ & 39,111 \\
\hline SPI049C & Doxorubicin & 30 & Suprateck & $\begin{array}{l}\text { A nonionic surfactant-based } \\
\text { micellar drug delivery system } \\
\text { comprising Pluronic }^{\circledR} \text { L6I and } \\
\text { FI27. This nanosystem has } \\
\text { demonstrated efficacy against } \\
\text { multidrug-resistant cells and is } \\
\text { currently in Phase III clinical trials. }\end{array}$ & 112,113 \\
\hline
\end{tabular}

Note: Alza Corporation (Palo Alto, CA, USA); NeXstar Pharmacutical (Boulder, CO, USA); Merck and Co., Inc. (Whitehouse Station, NJ, USA); American Pharmaceutical Partners Inc (Schuamburg, IL, USA); American Bioscience (Blauvelt, NY, USA); Nippon Kayaku (Tokyo, Japan); Samyang (Seoul, Korea); Supratek (Montreal, QC, Canada).

\section{Nanotheranostics}

Nanotheranostics, an area of research still in its infancy, refers to the simultaneous use of one nanosystem for diagnostic and therapeutic purposes. ${ }^{7,13,119}$ One of the primary benefits of these systems is individualization of therapeutic interventions. ${ }^{7,13}$ Therapeutic regimens can be designed and assessed according to the disease presentations of individual patients, thereby achieving optimized treatment outcomes with regard to safety and efficacy. ${ }^{7}$ Figure 6 summarizes the potential contribution of nanotheranostics to 


\section{Nanotheranostics}

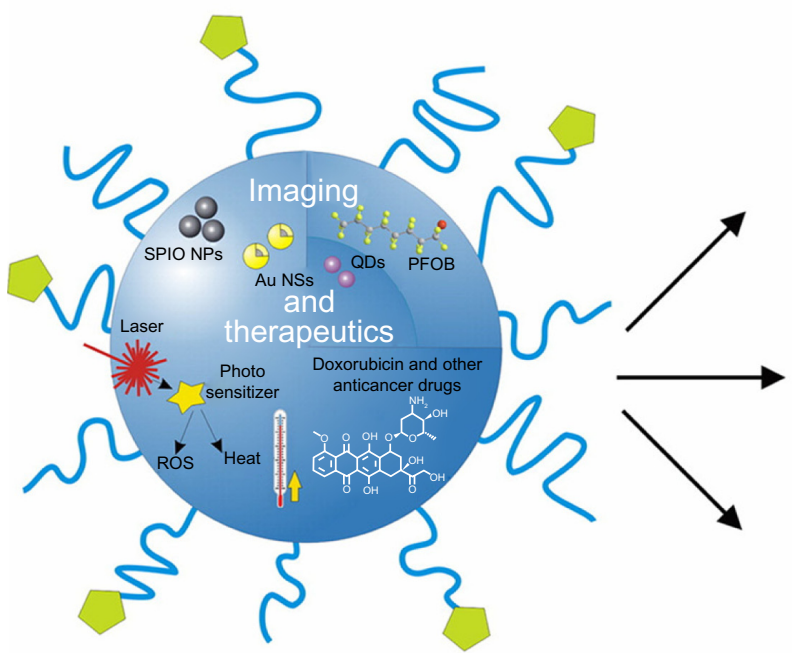

\section{Personalized nanomedicine}
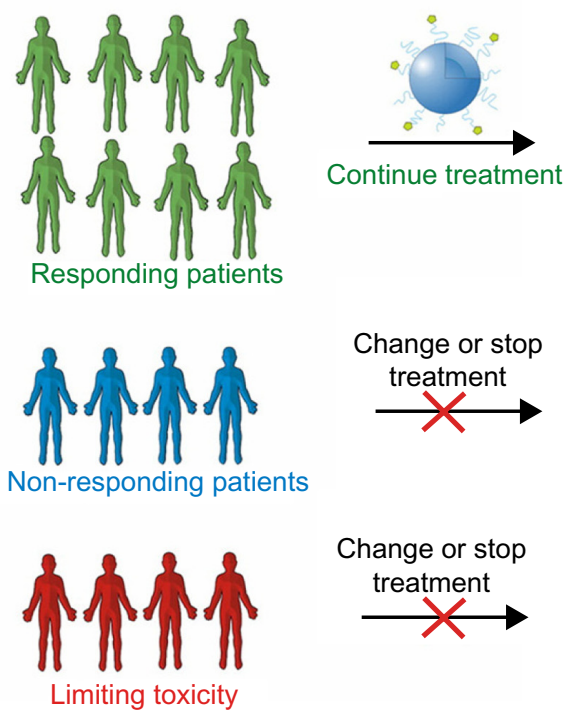

Change or stop treatment

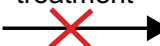

Change or stop treatment

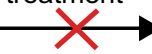

Figure 6 Illustration of the potential of nanotheranostic systems in personalizing treatment and improving therapeutic outcomes in cancer. ${ }^{120}$

Note: Reprinted from Advanced Drug Delivery Reviews, 64(I3), Mura S, Couvreur P, Nanotheranostics for personalized medicine, I394-I4I6, Copyright @ 20I2, with permission from Elsevier.

Abbreviations: SPIO, superparamagnetic iron oxide; NPs, nanoparticles; NSs, nanospheres; QDs, quantum dots; PFOB, perfluorooctylbromide; ROS, reactive oxygen species; Au, Gold.

individualization of cancer therapy and successful therapeutic outcomes. Invasive procedures can also be minimized by the combination of diagnosis and therapy. Moreover, the heterogeneous underpinnings of tumors will benefit tremendously from the individualization of therapy that nanotheranostic systems allow.

Various nanoconstructs have been created and investigated for potential theranostics application in solid tumors, such as liposomes, nanoparticles, micelles, and emulsions. ${ }^{121}$ Thus far, ultrasound has been largely investigated in combination with the nanoconstructs, as it allows for the safe and effective imaging of solid tumors as well as being capable of disrupting the nanoconstructs resulting in targeted drug release. ${ }^{121}$ Figure 7 presents a few nanoconfigurations formulated for theranostic applications. A graft terpolymer was employed by Shalviri et al to formulate self-assembling, DOX-containing nanoparticles, chelated with gadolinium, depicted schematically in Figure 7A. ${ }^{122}$ This innovative nanosystem demonstrates $\mathrm{pH}$-dependent drug release for targeted tumor therapy, as well as strong magnetic resonance imaging contrast for tumor imaging. Mura and Couvreur designed a nanoshell theranostic system with a dielectric silica core, surrounded by a thin gold (metal) shell and a second silica layer containing an imaging dye and iron oxide nanoparticles, illustrated in Figure 7B. ${ }^{120}$ This theranostic system has application in photothermal cancer therapy and contrast imaging through optical contrast tomography, through absorption and scattering, respectively, in the near-infrared region. A self-assembling mesoporous silica nanoparticle encapsulating carbon with silica nanocrystals embedded in the mesoporous wall is presented in Figure 7C. The system is conjugated to phospholipids and polysaccharides to create a theranostic system capable of simultaneously delivering antineoplastic drugs and luminescent imaging of tumor tissue in the near-infrared to visible region. ${ }^{123}$ Howell et al formulated a self-assembling lipid-micellar nanoparticle containing a DOX or DNA therapeutic payload and encapsulating a manganese core, illustrated in Figure 7D. ${ }^{124}$ This multifunctional nanosystem has application in contrast magnetic resonance imaging for tumor diagnosis, as well as in tumor therapy with conventional chemotherapeutic drugs or gene delivery. ${ }^{124}$

\section{Magnetic nanoparticles}

Magnetic nanoparticles (MNPs) have received considerable attention for application in the field of biomedical imaging and therapy, particularly with regard to cancer. ${ }^{54,119,125}$ At room temperature, these nanoparticles undergo strong magnetization in the presence of a magnetic field, which is reversed at the removal of the magnetic field. ${ }^{56}$ This allows for external manipulation to achieve a specific result, whether imaging of a specific region or body system or stimuli-responsive drug release. In addition, the high surface-area-to-volume ratio makes them ideal for the conjugation of targeting moieties to tailor the application. ${ }^{25}$ In vivo, biological barriers pose a significant challenge to the accessibility of imaging and drug delivery systems to 
A

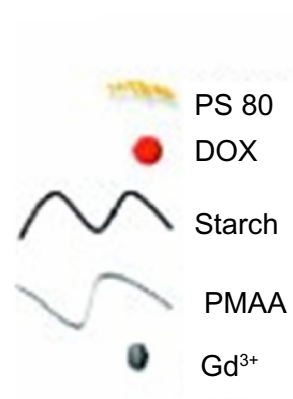

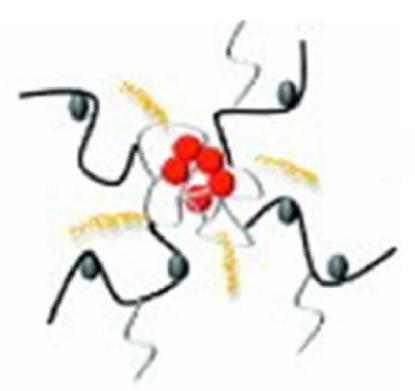

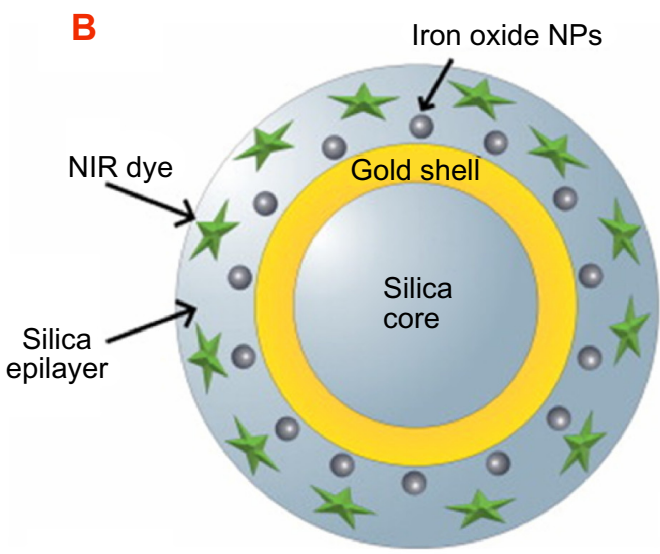

D

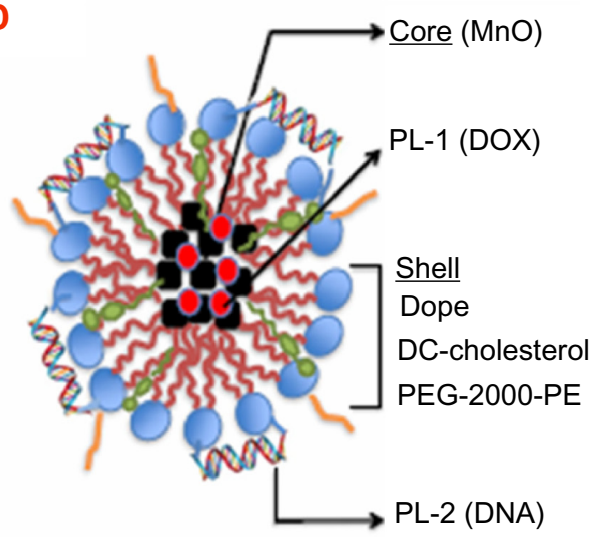

$P L-2(D N A)$
C

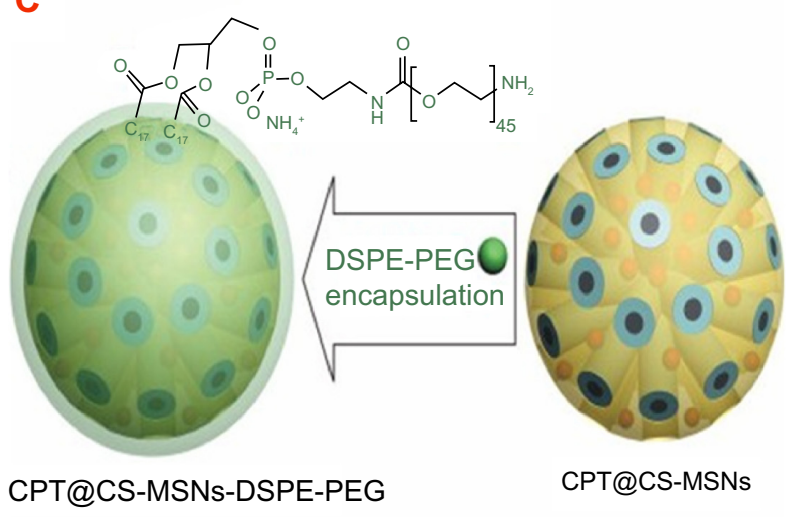

Figure 7 Innovative nanoconstructs with tumor theranostic application. (A) A self-assembling terpolymer nanoparticle containing doxorubicin. ${ }^{122}$ (B) A nanoshell theranostic system consisting of a dielectric silica core and a shell composed of an initial gold layer and a second layer of silica containing an imaging dye and iron oxide nanoparticles. ${ }^{120}$ (C) A self-assembling mesoporous nanoparticle encapsulating carbon, with silica nanocrystals embedded in the mesoporous nanoparticle wall. This nanosystem is further conjugated with phospholipids and polysaccharides. ${ }^{123}$ (D) A self-assembling lipid-micellar nanoparticle with a manganese core and either doxorubicin or DNA therapeutic agent. ${ }^{124}$

Notes: (A) Reprinted from Journal of Controlled Release, I67(I), Shalviri A, Foltz WD, Cai P, Rauth AM, Wu XY, Multifunctional terpolymeric MRI contrast agent with superior signal enhancement in blood and tumor, II-20, Copyright (C) 2013, with permission from Elsevier. (B) Reprinted from Advanced Drug Delivery Reviews, 64(I3), Mura S, Couvreur P, Nanotheranostics for personalized medicine, I394-14I6, Copyright (C) 2012, with permission from Elsevier. (C) Reprinted from Biomaterials, 33(I7), He Q, Ma M, Wei C, Shi J, Mesoporous carbon@silicon-silica nanotheranostics for synchronous delivery of insoluble drugs and luminescence imaging, 4392-4402, Copyright (C) 2012, with permission from Elsevier. (D) Reprinted from Journal of Controlled Release, 167(2), Howell M, Mallela J, Wang C, et al, Manganese-loaded lipid-micellar theranostics for simultaneous drug and gene delivery to lungs, 210-218, Copyright (C) 2013, with permission from Elsevier.

Abbreviations: PS 80, polysorbate 80; PMAA, poly(methacrylic acid); NIR, near infra red; Dope, dioleoylphosphatidyl-ethanolamine; DC, 33-[N-(N'-dimethylaminoethane)carbamoyl; PEG, polyethylene glycol; PE, phosphatidylethanolamine; PL-I, payload I; PL-2, payload 2; DSPE, distearoylphosphatidylethanolamine; MSNs, mesoporous silca nanoparticles; CS, carbon and silica nanocrystals; CPT, camptothecin; DOX, doxorubicin; NPs, nanoparticles.

the target site. ${ }^{119}$ MNPs are particularly adept at overcoming this challenge. The well-controlled narrow size range of MNP formulations allow them to traverse physiological barriers on their path to the intended site, aided by the application of the external stimulus. However, agglomeration resulting from dipole interaction of these nanoparticles poses a problem.

Several modifications have been investigated to overcome this problem, such as polymer coating. Moreover, the chosen polymers often had properties that were advantageous to the function of the magnetic nanoparticles, such as increasing circulation lifespan in vivo (PEG) and temperature- and pH-responsiveness (N-isopropylacrylamide, Chitosan). ${ }^{56}$ Modification of magnetic nanoparticles attempted by Rastogi et al included the use of thermosensitive polymers for hyperthermia-induced drug release, as well as folic acid conjugation for active tumor targeting. ${ }^{56}$ Magnetic resonance imaging conducted with the formulation attested to its applicability to in vivo use, whereas drug release was determined to be site-specific as a result of folic acid targeting, the nanosize capitalizing on the EPR effect, and an externally applied thermal stimulus.

Superparamagnetic iron oxide nanoparticles (SPIONs) are a popular subtype of magnetic nanoparticles that have been widely studied because of their targeting ability via the application of an external stimulus, as well as the favorable safety profile. ${ }^{119}$ SPIONs consist of an iron oxide core that is responsible for the magnetic targeting ability, which is either coated with a biocompatible polymer or is precipitated into the pores of a porous polymer. ${ }^{125}$ 
Superparamagnetic nanoparticles are localized by passive targeting as a result of their size, which is only applicable in certain conditions such as solid tumors; active targeting through the attachment of ligands; and the process of externally applied magnetic force overcoming the force exerted on the nanoparticles by blood flow. The nanoparticles are then able to extravasate out of the blood vessel and accumulate at the intended site of action, where imaging can be conducted or drug release can occur. ${ }^{125}$ SPION formulations of note are the commercially available Combidex ${ }^{\circledR}$ (Radboud University Nijmegen Medical Center, the Netherlands), which finds application in the imaging of lymph node metastasis, as well as Lumiren ${ }^{\circledR}$ (AMAG Pharmaceuticals Inc., Cambridge, MA, USA) for bowel imaging, which will be useful in the detection of colonic and colorectal. ${ }^{119}$

\section{PDT}

PDT involves the administration of a nontoxic agent that acts as a photosensitizer (PS). Photoexcitation of the PSs leads to the generation of free radicals, which destroy tumor tissue. ${ }^{126.127}$ An alternative method of PDT involves the transition of the PS from a singlet to an excited triplet state. Transfer of energy from the excited PS to oxygen molecules results in the production of singlet oxygen species, which destroy cellular components, causing cell death, as illustrated in Figure $8 .{ }^{127}$ Subsequently, tumors are irradiated with light of a predetermined wavelength, which ultimately causes the generation of cytotoxic products through the interaction of the PS and light in the presence of oxygen. ${ }^{7}$ These cytotoxic products result in tissue destruction and cell death. ${ }^{66}$ The chosen wavelength of irradiating light should ideally be one that is absorbed to a greater degree by the tumor tissue relative to the surrounding healthy tissue.

PSs have been widely investigated for application in cancer imaging and therapy. ${ }^{7}$ Table 3 draws attention to nanosystems that have demonstrated potential for use in PDT of various cancer subtypes. One such PS is hypericin, which has gained attention for its potential in the diagnosis of bladder and oral tumors in addition to its combination with bevacizumab for the treatment of bladder cancer. ${ }^{7}$ When combined with other imaging techniques, PDT can even be used as an aid in cytoreductive surgery. However, the potential for clinical application of these agents is marred by pharmacokinetic problems such as nonselective distribution of PSs, which may prove dangerous in highly disseminated tumors, as is often the case with ovarian, colon, and pancreatic tumors. ${ }^{66}$ The hydrophobic nature of most PSs result in a tendency to aggregate in biological environments, which alters the optical properties of PSs, as well as oxygen production. ${ }^{66}$ The solubility profile poses challenges to the delivery of PSs. ${ }^{7}$ PSs display suboptimal tissue half-life in vivo, as well as the possibility of toxicity in the absence of irradiation (ie, dark toxicity). ${ }^{128}$ PSs exhibit a limitation on the targeting depth resulting from attenuated intensity of irradiation after propagation through skin and tissue. ${ }^{128}$

It is the above-mentioned shortcomings that have triggered the emergence of nanoparticulate-based PDT. Formulation of PSs in a nanosystem can greatly influence its activity by altering the pharmacokinetic properties, localization, and cellular uptake of the PS. ${ }^{129}$ Selectivity for tumor tissue is pivotal to the safety and efficacy of PDT. As previously highlighted, the preferential distribution of nanosystems into tumor tissue is advantageous as a method of targeting.

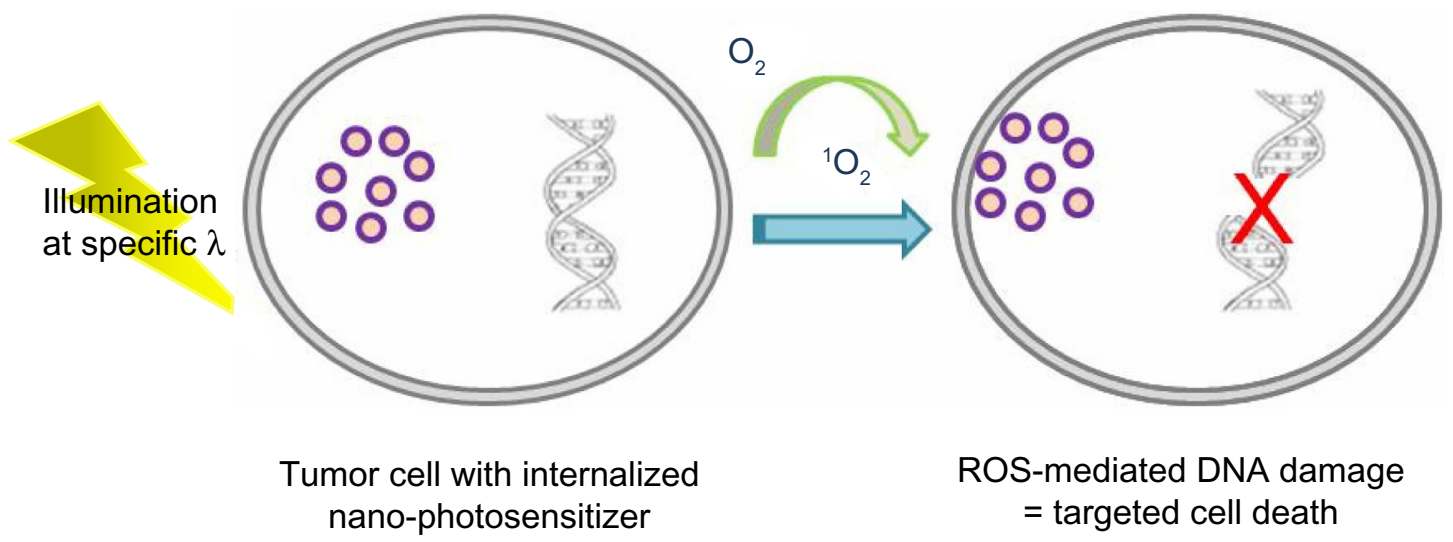

Figure 8 Illustrative representation of the mechanism of photosensitizers for photodynamic therapy. ${ }^{127}$

Note: Reprinted from Cancer Letters, 327(I-2), Spyratou E, Makropoulou M, Mourelatou EA, Demetzos C, Biophotonic techniques for manipulation and characterization of drug delivery nanosystems in cancer therapy, III-122, Copyright (c) 2012, with permission from Elsevier. Abbreviation: ROS, reactive oxygen species. 
Table 3 Nanosystems investigated for use in photodynamic therapy, with therapeutic and/or imaging potential

\begin{tabular}{|c|c|c|c|c|c|}
\hline Nanosystem & $\begin{array}{l}\text { Imaging } \\
\text { potential }\end{array}$ & $\begin{array}{l}\text { Therapeutic } \\
\text { potential }\end{array}$ & $\begin{array}{l}\text { Type of } \\
\text { study }\end{array}$ & Features & References \\
\hline $\begin{array}{l}\text { Mesoporous silica } \\
\text { nanoparticles }\end{array}$ & $x$ & $x$ & $\begin{array}{l}\text { In vitro, } \\
\text { ex vivo }\end{array}$ & $\begin{array}{l}\text { Drug and imaging molecules can be incorporated into the } \\
\text { pores of the nanoparticles. In vitro studies demonstrated } \\
\text { successful tumor cell uptake and cytotoxicity. }\end{array}$ & 132 \\
\hline $\begin{array}{l}\text { Foslip }^{\circledR} \text {, liposomal } \\
\text { preparation }\end{array}$ & & $x$ & $\begin{array}{l}\text { In vitro, } \\
\text { ex vivo }\end{array}$ & $\begin{array}{l}\text { Liposomal preparation containing Foscan (temoporfin). } \\
\text { Has exhibited enhanced efficacy and reduced dark } \\
\text { toxicity relative to Foscan. }\end{array}$ & 65,133 \\
\hline $\begin{array}{l}\text { Zinc(II)phthalocyanine- } \\
\text { containing micelles }\end{array}$ & $x$ & $x$ & In vitro & $\begin{array}{l}\text { Micellar formulation proved more stable with an } \\
\text { enhanced lifetime of the triplet oxygen excited state } \\
\text { (cytotoxic), augmented fluorescence quantum yield, and } \\
\text { protection of the hydrophobic compound zinc(II) } \\
\text { phthalocyanine. }\end{array}$ & 134 \\
\hline $\begin{array}{l}\text { Peptide-coated quantum } \\
\text { dots conjugated to } \\
\text { photosensitizers }\end{array}$ & $x$ & $x$ & In vitro & $\begin{array}{l}\text { Water-soluble quantum dot that retains the } \\
\text { photophysical properties of the quantum dot and } \\
\text { photosensitizer. Efficient generation of singlet oxygen } \\
\text { through fluorescence resonance energy transfer. }\end{array}$ & 125 \\
\hline $\begin{array}{l}\text { Aerosol OT-alginate } \\
\text { nanoparticles containing } \\
\text { doxorubicin and } \\
\text { photosensitizer } \\
\text { methylene blue }\end{array}$ & & $x$ & $\begin{array}{l}\text { In vitro, } \\
\text { ex vivo }\end{array}$ & $\begin{array}{l}\text { Combination of photodynamic therapy with chemotherapy } \\
\text { by incorporating methylene blue, a photosensitizer, into } \\
\text { doxorubicin-containing nanoparticles displayed enhanced } \\
\text { in vitro cytotoxicity in a resistant tumor cell line. }\end{array}$ & 135 \\
\hline $\begin{array}{l}\text { Pullulan-folate-coated } \\
\text { lipid nanoparticles }\end{array}$ & & $x$ & $\begin{array}{l}\text { In vitro, } \\
\text { ex vivo }\end{array}$ & $\begin{array}{l}\text { Pullulan-folate-coated lipid nanoparticles demonstrated } \\
\text { enhanced nuclei targeting and accumulation, the desired } \\
\text { site for enhanced photodynamic therapy efficacy, and } \\
\text { enhanced efficacy at low-intensity irradiation, for treatment } \\
\text { of deep-seated tumors. In addition, doxorubicin substitution } \\
\text { highlighted the versatility of the system, enhancing the } \\
\text { already present nuclei accumulation of doxorubicin. }\end{array}$ & 130 \\
\hline $\begin{array}{l}\text { Organically modified } \\
\text { silica nanoparticles }\end{array}$ & & $x$ & $\begin{array}{l}\text { In vitro, } \\
\text { ex vivo, } \\
\text { in vivo }\end{array}$ & $\begin{array}{l}\text { Organically modified silica nanoparticles covalently } \\
\text { linked to a photosensitizer, to avoid release of the } \\
\text { photosensitizer in systemic circulation. Spectroscopic and } \\
\text { functional properties were retained by this system. This } \\
\text { system has the potential for bifunctional diagnostic and } \\
\text { therapeutic value with slight modification. }\end{array}$ & 136 \\
\hline
\end{tabular}

Notes: $\mathrm{X}=$ yes. Foslip ${ }^{\circledast}$ (Sanofi-Aventis, Paris, France).

Furthermore, nanosystems are amenable to the attachment of targeting moieties, which will facilitate a greater targeting capacity. Incorporation of the hydrophobic PSs into nanosystems that exhibit outward hydrophilicity will stabilize the formulation in physiological environments, as well as minimize the challenges associated with administration. Table 3 highlights a few examples of nanosystems that have been investigated for PDT.

An investigation undertaken by García-Díaz et al succinctly illustrates the unprecedented influence nanotechnological manipulation may have on the effectiveness of PDT in cancer therapy. ${ }^{129}$ Determining the in vitro and in vivo activity of novel photosensitizer, temocene, in relation to drug delivery vehicles containing the active molecule, this team was able to demonstrate the enhanced safety and in vivo activity of temocene incorporated into liposomes and micelles. ${ }^{129}$ This work also emphasized the stability of liposomal and micelle drug delivery vehicles, with respect to lyophilization and aggregation. A highlight of this study to be noted was the toxic effects of free temocene in vivo, as a result of vascular aggregation of the PS. This resulted in the discontinuation of in vivo studies on free temocene. Liposome-incorporated temocene, however, demonstrated the greatest tumor selectivity and cell internalization. Micellar temocene, although lacking in activity in vitro, was the most effective in vivo when combined with a short drug-to-light interval, as a result of the targeting of tumor vasculature that is absent in cell cultures used for in vitro analysis. ${ }^{129}$

In another approach dedicated to colorectal cancer, researchers at the University of Buffalo in New York, NY, USA (part of the National Cancer Institute Alliance for Nanotechnology in Cancer), and scientists at the Roswell Park Cancer Institute, also in Buffalo, use a silica-based nanoshell that encases a PS. ${ }^{22}$ They report ready uptake 
of these nanoshells by the laboratory-grown tumor cells. On exposure to light of a given wavelength, the PS within the cells gets activated, triggering the production of reactive oxygen molecules that ultimately kill the cancer cells. The technology is under clinical trials for safety. The same researchers are further developing a second-generation PS-loaded nanoshell that incorporates tumor-targeting and imaging agents to deliver tumor-specific, image-guided therapy for cancer. ${ }^{22}$

Although PDT has become a widely accepted diagnostic and therapeutic mechanism for certain tumors, there remains some challenges with regard to larger and more deeply located tumors; namely, the dependence of PDT efficacy on light dose, which is compromised for larger and deeper tumors. ${ }^{130}$ Because the mechanism of action of the singlet oxygen generated in PDT is DNA damage and inactivation of enzymes responsible for DNA repair, the logical cellular target for PSs in PDT would be the nucleus. ${ }^{130,131}$ The generation of these cytotoxic singlet oxygen species beyond the cell and the ultimate target, the nucleus, leads to reduced efficacy resulting from the short diffusion range of the singlet oxygen. ${ }^{130}$ Hence, subcellular delivery, distribution, and accumulation of the PS is fundamental to the efficacy of PDT. ${ }^{131}$ To address this challenge, Ling et al formulated a lipid nanoparticle coated with folate-containing pullulan, which would be stable in plasma and targeted to the overexpressed folate receptor on tumor cells, thereby accumulating preferentially in, and be endocytosed into, tumor cells. ${ }^{130}$ Enzymatic degradation of the pullulan-folate coating releases lipid and drug, which form lipid-drug complexes, the lipophilicity of which facilitates transport into the nucleus of the cell. A focal point of this study was the demonstration of enhanced nuclei accumulation of the model and other drugs, indicative of the potential for enhanced PDT that is more widely applicable in clinical cancer therapy, as well as the versatility of this nanosystem. Furthermore, augmented cytotoxicity was exhibited at low irradiation intensity, highlighting its applicability to the treatment of deeply located tumors. ${ }^{130}$

\section{Conclusion}

Successful nanotechnological innovations that transcend medical ideologies decades ahead of time demand the amalgamation of several individual specialties including, but not limited to, biology, chemistry, engineering, mathematics, and pharmacology. Nanoscale dimensions have demonstrated characteristics that are vastly different from identical compounds at the micro- and macroscale.
Exploitation of these characteristics has been extensively investigated for application in the detection and treatment of various solid tumors. The potential of nanotechnology highlighted during these studies brings forth innovations that could revolutionize the incidence, prognosis, mortality, and burden of cancer. The nanotechnology industry is growing exponentially, with billions being invested in research and development. The returns are, undoubtedly, expected to far surpass the investment, and the medical field appears to be a forerunner in nanotechnological innovations.

The escalating incidence of cancer worldwide and the increased exposure to carcinogens necessitates radical advancements in cancer detection and treatment to improve therapeutic outcomes, as well as reduce the burden on health care systems. Hence, this disease state has dominated nanotechnological investigations. Nanosystems have exhibited potential at the pathophysiological level, in terms of passive targeting to tumor tissue, transportation into tumor cells and bypassing mechanisms of tumor resistance, as well as at the biopharmaceutical level, by enhancing drug solubilization, efficacy, and hence clinical relevance. In addition, nanosystems have proven to be immensely feasible in addressing the shortfalls of therapeutically active phytochemicals, whose cost-effectiveness and ease of procurement render them invaluable treatment options.

Commercially available nanosystems, as well as those in late-stage clinical trials, validate the feasibility of research in the field of nanotechnology and cancer. Moreover, the development of drug delivery systems to improve the pharmacokinetics, biodistribution, bioavailability, and safety profiles of existing drug molecules is particularly attractive, as it is usually a shorter, more economical process relative to that involved in the identification and registration of new drug compounds. Nanosystems elevate the paradigm of targeted therapy to another echelon, surpassed only by the potential to combine tumor detection, therapy, and monitoring mechanisms into one system. Although the current outlook on the role of nanotechnology in addressing the cancer detection and treatment shortfalls that plague medical experts appears to be immensely promising, our understanding on the mechanisms, fate, and effect of nanosystems and their development is far from absolute. These issues will need to be intensely investigated for the responsible development of widely employed nanosystems to thrive. With that said, we may indeed be only a few nanometers away from comprehensive, multimodal solutions to the "cancer plague." 


\section{Acknowledgments}

The study was funded by the National Research Foundation of South Africa.

\section{Disclosure}

The authors report no conflicts of interest in this work.

\section{References}

1. World Health Organization. Cancer Fact Sheet No 297. Geneva: World Health Organization; 2013. Available from: http://www.who.int/mediacentre/factsheets/fs297/en/index.html. Accessed January 31, 2013.

2. McCormack VA, Schüz J. Africa's growing cancer burden: environmental and occupational contributions. Cancer Epidemiol. 2012;36(1):1-7.

3. Ferlay J, Shin HR, Bray F, Forman D, Mathers C and Parkin DM. GLOBOCAN 2008 v2.0, Cancer Incidence and Mortality Worldwide: IARC CancerBase No. 10 [Internet]. Lyon, France: International Agency for Research on Cancer; 2010. Available from: http://globocan.iarc.fr. Accessed 30, October 2013.

4. National Cancer Institute. What Is Cancer? Defining Cancer. Bethesda, MD: National Cancer Institute. Available from: http://www. cancer.gov/cancertopics/cancerlibrary/what-is-cancer. Accessed February 28, 2013.

5. Ramasamy K, Agarwal R. Multitargeted therapy of cancer by silymarin. Cancer Lett. 2008;269(2):352-362.

6. Khosravi-Darani K, Pardakhty A, Honarpisheh H, Rao VS, Mozafari MR. The role of high-resolution imaging in the evaluation of nanosystems for bioactive encapsulation and targeted nanotherapy. Micron. 2007;38(8):804-818.

7. Rai P, Mallidi S, Zheng X, et al. Development and applications of photo-triggered theranostic agents. Adv Drug Deliv Rev. 2010;62(11): 1094-1124.

8. Bawarski WE, Chidlowsky E, Bharali DJ, Mousa SA. Emerging nanopharmaceuticals. Nanomedicine. 2008;4(4):273-282.

9. Etheridge ML, Campbell SA, Erdman AG, Haynes CL, Wolf SM, McCullough J. The big picture on nanomedicine: the state of investigational and approved nanomedicine products. Nanomedicine. 2013;9(1): 1-14.

10. Farokhzad OC, Langer R. Nanomedicine: developing smarter therapeutic and diagnostic modalities. Adv Drug Deliv Rev. 2006;58(14): $1456-1459$.

11. Dominguez AL, Lustgarten J. Targeting the tumor microenvironment with anti-neu/anti-CD40 conjugated nanoparticles for the induction of antitumor immune responses. Vaccine. 2010;28(5):1383-1390.

12. Chen K-I, Li B-R, Chen Y-T. Silicon nanowire field-effect transistorbased biosensors for biomedical diagnosis and cellular recording investigation. Nano Today. 2011;6(2):131-154.

13. Vizirianakis IS. Nanomedicine and personalized medicine toward the application of pharmacotyping in clinical practice to improve drugdelivery outcomes. Nanomedicine. 2011;7(1):11-17.

14. Ji XT, Huang L, Huang HQ. Construction of nanometer cisplatin core-ferritin (NCC-F) and proteomic analysis of gastric cancer cell apoptosis induced with cisplatin released from the NCC-F. J Proteomics. 2012;75(11):3145-3157.

15. Chen H, Wang L, Yeh J, et al. Reducing non-specific binding and uptake of nanoparticles and improving cell targeting with an antifouling PEO-b-PgammaMPS copolymer coating. Biomaterials. 2010;31(20): $5397-5407$.

16. Ranganathan R, Madanmohan S, Kesavan A, et al. Nanomedicine: towards development of patient-friendly drug-delivery systems for oncological applications. Int J Nanomedicine. 2017:1043-1060.

17. Jabir NR, Tabrez S, Ashraf GM, Shakil S, Damanhouri GA, Kamal MA. Nanotechnology-based approaches in anticancer research. Int $J$ Nanomedicine. 2017:4391-4408.
18. Fishman DA, Cohen L, Blank SV, et al. The role of ultrasound evaluation in the detection of early-stage epithelial ovarian cancer. Am J Obstet Gynecol. 2005;192(4):1214-1221.

19. Wu X, Jiang H, Zheng J, et al. Highly sensitive recognition of cancer cells by electrochemical biosensor based on the interface of gold nanoparticles/polylactide nanocomposites. J Electroanal Chem. 2011; 656(1-2):174-178.

20. Vo-Dinh T, Cullum BM, Stokes DL. Nanosensors and biochips: frontiers in biomolecular diagnostics. Sens Actuators. 2001;74(1-3):2-11.

21. Kwon OS, Park SJ, Jang J. A high-performance VEGF aptamer functionalized polypyrrole nanotube biosensor. Biomaterials. 2010;31(17): 4740-4747.

22. National Institutes of Health. Advances in Colorectal Cancer Research: Nanotechnology to Improve Early Detection and Treatment of Colorectal Cancer. Bethesda, MD: National Institutes of Health. Available from: http://www.nih.gov/science/colorectalcancer/nanotech.htm. Accessed May 31, 2013.

23. Zheng XT, Li CM. Single living cell detection of telomerase overexpression for cancer detection by an optical fiber nanobiosensor. Biosens Bioelectron. 2010;25(6):1548-1552.

24. Geszke M, Murias M, Balan L, et al. Folic acid-conjugated core/ shell $\mathrm{ZnS}: \mathrm{Mn} / \mathrm{ZnS}$ quantum dots as targeted probes for two photon fluorescence imaging of cancer cells. Acta Biomater. 2011;7(3): 1327-1338.

25. Schroeder JE, Shweky I, Shmeeda H, Banin U, Gabizon A. Folate-mediated tumor cell uptake of quantum dots entrapped in lipid nanoparticles. J Control Release. 2007;124(1-2):28-34.

26. Pan J, Feng SS. Targeting and imaging cancer cells by folate-decorated, quantum dots (QDs)-loaded nanoparticles of biodegradable polymers. Biomaterials. 2009;30(6):1176-1183.

27. Papagiannaros A, Levchenko T, Hartner W, Mongayt D, Torchilin V. Quantum dots encapsulated in phospholipid micelles for imaging and quantification of tumors in the near-infrared region. Nanomedicine. 2009;5(2):216-224.

28. Siddiqui IA, Adhami VM, Chamcheu JC, Mukhtar H. Impact of nanotechnology in cancer: emphasis on nanochemoprevention. Int $J$ Nanomedicine. 2012;7:591-605.

29. Setua S, Menon D, Asok A, Nair S, Koyakutty M. Folate receptor targeted, rare-earth oxide nanocrystals for bi-modal fluorescence and magnetic imaging of cancer cells. Biomaterials. 2010;31(4):714-729.

30. Chin PT, Buckle T, Aguirre de Miguel A, Meskers SC, Janssen RA, van Leeuwen FW. Dual-emissive quantum dots for multispectral intraoperative fluorescence imaging. Biomaterials. 2010;31(26):6823-6832.

31. Kim PS, Djazayeri S, Zeineldin R. Novel nanotechnology approaches to diagnosis and therapy of ovarian cancer. Gynecol Oncol. 2011;120(3): 393-403.

32. Cirstoiu-Hapca A, Buchegger F, Lange N, Bossy L, Gurny R, Delie F. Benefit of anti-HER2-coated paclitaxel-loaded immuno-nanoparticles in the treatment of disseminated ovarian cancer: Therapeutic efficacy and biodistribution in mice. J Control Release. 2010;144(3): 324-331.

33. Lei T, Srinivasan S, Tang Y, et al. Comparing cellular uptake and cytotoxicity of targeted drug carriers in cancer cell lines with different drug resistance mechanisms. Nanomedicine. 2011;7(3):324-332.

34. Guyton AC. Textbook of Medical Physiology. 8th ed. San Diego: Harcourt College Publishers; 1991:274.

35. Merisko-Liversidge E, Liversidge GG, Cooper ER. Nanosizing: a formulation approach for poorly-water-soluble compounds. Eur $J$ Pharm Sci. 2003;18(2):113-120.

36. Beig A, Miller JM, Dahan A. Accounting for the solubility-permeability interplay in oral formulation development for poor water solubility drugs: the effect of PEG-400 on carbamazepine absorption. Eur J Pharm Biopharm. 2012;81(2):386-391.

37. Buckley ST, Fischer SM, Fricker G, Brandl M. In vitro models to evaluate the permeability of poorly soluble drug entities: challenges and perspectives. Eur J Pharm Sci. 2012;45(3):235-250. 
38. Savic R, Luo L, Eisenberg A, Maysinger D. Micellar nanocontainers distribute to defined cytoplasmic organelles. Science. 2003;300(5619): 615-618.

39. Pathak P, Meziani MJ, Desai T, et al. Formation and stabilization of ibuprofen nanoparticles in supercritical fluid processing. J Supercrit Fluids. 2006;37(3):279-286.

40. Lu Y, Park K. Polymeric micelles and alternative nanonized delivery vehicles for poorly soluble drugs. Int J Pharm. 2013;453(1): 198-214.

41. Ganta S, Paxton JW, Baguley BC, Garg S. Formulation and pharmacokinetic evaluation of an asulacrine nanocrystalline suspension for intravenous delivery. Int J Pharm. 2009;367(1-2):179-186.

42. Ehrlich P. Collected Studies on Immunity. New York: John Wiley; 1906:442.

43. Rapoport N. Physical stimuli-responsive polymeric micelles for anticancer drug delivery. Prog Polym Sci. 2007;32:962-990.

44. Hernot S, Klibanov AL. Microbubbles in ultrasound-triggered drug and gene delivery. Adv Drug Deliv Rev. 2008;60(10):1153-1166.

45. Husseini GA, Pitt WG. Micelles and nanoparticles for ultrasonic drug and gene delivery. Adv Drug Deliv Rev. 2008;60(10):1137-1152.

46. Shapira A, Livney YD, Broxterman HJ, Assaraf YG. Nanomedicine for targeted cancer therapy: towards the overcoming of drug resistance. Drug Resist Updat. 2011;14(3):150-163.

47. Cho YW, Park SA, Han TH, et al. In vivo tumor targeting and radionuclide imaging with self-assembled nanoparticles: mechanisms, key factors, and their implications. Biomaterials. 2007;28(6): 1236-1247.

48. Geusens B, Lambert J, De Smedt SC, Buyens K, Sanders NN, Van Gele M. Ultradeformable cationic liposomes for delivery of small interfering RNA (siRNA) into human primary melanocytes. J Control Release. 2009;133(3):214-220.

49. Brannon-Peppas L, Blanchette JO. Nanoparticle and targeted systems for cancer therapy. Adv Drug Deliv Rev. 2004;56(11): 1649-1659.

50. Guo M, Que C, Wang C, Liu X, Yan H, Liu K. Multifunctional superparamagnetic nanocarriers with folate-mediated and $\mathrm{pH}$-responsive targeting properties for anticancer drug delivery. Biomaterials. 2011;32(1):185-194.

51. Blagosklonny MV. Matching targets for selective cancer therapy. Drug Discov Today. 2003;8(24):1104-1107.

52. Lehner R, Wang X, Wolf M, Hunziker P. Designing switchable nanosystems for medical application. J Control Release. 2012;161(2): 307-316.

53. Meng $\mathrm{F}$, Cheng $\mathrm{R}$, Deng $\mathrm{C}$, et al. Intracellular drug release nanosystems. Materials Today. 2012;15(10):436-442.

54. Leamon CP, Low PS. Folate-mediated targeting: from diagnostics to drug and gene delivery. Drug Discov Today. 2001;6(1):44-51.

55. Vauthier C, Dubernet C, Chauvierre C, Brigger I, Couvreur P. Drug delivery to resistant tumors: the potential of poly(alkyl cyanoacrylate) nanoparticles. J Control Release. 2003;93(2):151-160.

56. Rastogi R, Gulati N, Kotnala RK, Sharma U, Jayasundar R, Koul V. Evaluation of folate conjugated pegylated thermosensitive magnetic nanocomposites for tumor imaging and therapy. Colloids Surf B Biointerfaces. 2011;82(1):160-167.

57. Pradhan P, Giri J, Rieken F, et al. Targeted temperature sensitive magnetic liposomes for thermo-chemotherapy. J Control Release. 2010;142(1):108-121.

58. Paolino D, Licciardi M, Celia C, Giammona G, Fresta M, Cavallaro G. Folate-targeted supramolecular vesicular aggregates as a new frontier for effective anticancer treatment in in vivo model. Eur J Pharm Biopharm. 2012;82(1):94-102.

59. Wang X, Li J, Wang Y, et al. HFT-T, a targeting nanoparticle, enhances specific delivery of paclitaxel to folate receptor-positive tumors. ACS Nano. 2009;3(10):3165-3174.

60. Wang X, Li J, Wang Y, et al. A folate receptor-targeting nanoparticle minimizes drug resistance in a human cancer model. ACS Nano. 2011;5(8):6184-6194.
61. Onyüksel H, Mohanty PS, Rubinstein I. VIP-grafted sterically stabilized phospholipid nanomicellar 17-allylamino-17-demethoxy geldanamycin: a novel targeted nanomedicine for breast cancer. Int $J$ Pharm. 2009;365(1-2):157-161.

62. Landriscina M, Amoroso MR, Piscazzi A, Esposito F. Heat shock proteins, cell survival and drug resistance: the mitochondrial chaperone TRAP1, a potential novel target for ovarian cancer therapy. Gynecol Oncol. 2010;117(2):177-182.

63. Mi Y, Liu X, Zhao J, Ding J, Feng SS. Multimodality treatment of cancer with herceptin conjugated, thermomagnetic iron oxides and docetaxel loaded nanoparticles of biodegradable polymers. Biomaterials. 2012;33(30):7519-7529.

64. Bazell R. HER-2: The making of Herceptin, a revolutionary treatment for breast cancer. New York: Random House; 1998.

65. Das M, Sahoo SK. Epithelial cell adhesion molecule targeted nutlin3a loaded immunonanoparticles for cancer therapy. Acta Biomater. 2011;7(1):355-369.

66. Paszko E, Ehrhardt C, Senge MO, Kelleher DP, Reynolds JV. Nanodrug applications in photodynamic therapy. Photodiagnosis Photodyn Ther. 2011;8(1):14-29.

67. Li X, Zhao Q, Qiu L. Smart ligand: Aptamer-mediated targeted delivery of chemotherapeutic drugs and siRNA for cancer therapy. J Control Release. 2013;171(2):152-162.

68. Soundararajan S, Wang L, Sridharan V, et al. Plasma membrane nucleolin is a receptor for the anticancer aptamer AS1411 in MV4-11 leukemia cells. Mol Pharmacol. 2009;76(5):984-991.

69. Yang X, Liu X, Liu Z, Pu F, Ren J, Qu X. Near-infrared light-triggered, targeted drug delivery to cancer cells by aptamer gated nanovehicles. Adv Mater. 2012;24(21):2890-2895.

70. Kang H, O’Donoghue MB, Liu H, Tan W. A liposome-based nanostructure for aptamer directed delivery. Chem Commun (Camb). 2010;46(2): 249-251.

71. Platt VM, Szoka FC Jr. Anticancer therapeutics: targeting macromolecules and nanocarriers to hyaluronan or CD44, a hyaluronan receptor. Mol Pharm. 2008;5(4):474- 486.

72. Ganesh S, Iyer AK, Morrissey DV, Amiji MM. Hyaluronic acid based self-assembling nanosystems for CD44 target mediated siRNA delivery to solid tumors. Biomaterials. 2013;34(13):3489-3502.

73. Gary-Bobo M, Brevet D, Benkirane-Jessel N, et al. Hyaluronic acid-functionalized mesoporous silica nanoparticles for efficient photodynamic therapy of cancer cells. Photodiagnosis Photodyn Ther. 2012;9(3):256-260.

74. Lo YL. Phospholipids as multidrug resistance modulators of the transport of epirubicin in human intestinal epithelial Caco-2 cell layers and everted gut sacs of rats. Biochem Pharmacol. 2000;60(9): 1381-1390.

75. Gao Z, Zhang L, Sun Y. Nanotechnology applied to overcome tumor drug resistance. J Control Release. 2012;162(1):45-55.

76. Win KY, Feng S-S. Effect of particle size and surface coating on cellular uptake of polymeric nanoparticles for oral delivery of anticancer drugs. Biomaterials. 2005;26(15):2713-2722.

77. Dreis S, Rothweiler F, Michaelis M, Cinatl J Jr, Kreuter J, Langer K. Preparation, characterisation and maintenance of drug efficacy of doxorubicin-loaded human serum albumin (HSA) nanoparticles. Int $J$ Pharm. 2007;341(1-2):207-214.

78. Wartlick H, Michaelis K, Balthasar S, Strebhardt K, Kreuter J, Langer K. Highly specific HER2-mediated cellular uptake of antibody-modified nanoparticles in tumour cells. J Drug Target. 2004;12(7):461-471.

79. Ertl B, Platzer P, Wirth M, Gabor F. Poly(D,L-lactic-co-glycolic acid) microspheres for sustained delivery and stabilization of camptothecin. J Control Release. 1999;61(3):305-317.

80. Mu L, Elbayoumi TA, Torchilin VP. Mixed micelles made of poly(ethylene glycol)-phosphatidylethanolamine conjugate and d-alpha-tocopheryl polyethylene glycol 1000 succinate as pharmaceutical nanocarriers for camptothecin. Int J Pharm. 2005;306(1-2):142-149. 
81. Koo OM, Rubinstein I, Onyuksel H. Camptothecin in sterically stabilized phospholipid micelles: a novel nanomedicine. Nanomedicine. 2005;1(1):77-84

82. Fang JY, Hung CF, Hua SC, Hwang TL. Acoustically active perfluorocarbon nanoemulsions as drug delivery carriers for camptothecin: drug release and cytotoxicity against cancer cells. Ultrasonics. 2009;49(1): 39-46.

83. Shenderova A, Burke TG, Schwendeman SP. Stabilization of 10-hydroxycamptothecin in poly(lactide-co-glycolide) microsphere delivery vehicles. Pharm Res. 1997;14(10):1406-1414.

84. Zhang L, Yang M, Wang Q, et al. 10-Hydroxycamptothecin loaded nanoparticles: preparation and antitumor activity in mice. $J$ Control Release. 2007;119(2):153-162.

85. Hatefi A, Amsden B. Camptothecin delivery methods. Pharm Res. 2002;19(10):1389-1399.

86. Ziomkowska B, Kruszewski S, Siuda R, et al. Deactivation of camptothecin determined by factor analysis of steady-state flourescence and absorption spectra. Optica Applicata. 2006;36(1):137-146.

87. Cortesi R, Esposito E, Maietti A, Menegatti E, Nastruzzi C. Formulation study for the antitumour drug camptothecin: liposomes, micellar solutions and a microemulsion. Int J Pharm. 1997;159(1):95-103.

88. Miura H, Onishi H, Sasatsu M, Machida Y. Antitumor characteristics of methoxypolyethylene glycol-poly(DL-lactic acid) nanoparticles containing camptothecin. J Control Release. 2004;97(1):101-113.

89. Vergara D, Bellomo C, Zhang X, et al. Lapatinib/Paclitaxel polyelectrolyte nanocapsules for overcoming multidrug resistance in ovarian cancer. Nanomedicine. 2012;8(6):891-899.

90. Fonseca C, Simões S, Gaspar R. Paclitaxel-loaded PLGA nanoparticles: preparation, physicochemical characterization and in vitro anti-tumoral activity. J Control Release. 2002;83(2):273-286.

91. Sonnemann J, Palani CD, Wittig S, et al. Anticancer effects of the p53 activator nutlin-3 in Ewing's sarcoma cells. Eur J Cancer. 2011;47(9): 1432-1441.

92. Song XR, Cai Z, Zheng Y, et al. Reversion of multidrug resistance by co-encapsulation of vincristine and verapamil in PLGA nanoparticles. Eur J Pharm Sci. 2009;37(3-4):300-305.

93. Cesur H, Rubinstein I, Pai A, Onyüksel H. Self-associated indisulam in phospholipid-based nanomicelles: a potential nanomedicine for cancer. Nanomedicine. 2009;5(2):178-183.

94. Mohanty C, Sahoo SK. The in vitro stability and in vivo pharmacokinetics of curcumin prepared as an aqueous nanoparticulate formulation. Biomaterials. 2010;31(25):6597-6611.

95. Das RK, Kasoju N, Bora U. Encapsulation of curcumin in alginatechitosan-pluronic composite nanoparticles for delivery to cancer cells. Nanomedicine. 2010;6(1):153-160.

96. Anitha A, Maya S, Deepa N, et al. Efficient water soluble O-carboxymethyl chitosan nanocarrier for the delivery of curcumin to can cells. Carbohydr Polym. 2011;83(2):452-461.

97. Tiyaboonchai W, Tungpradit W, Plianbangchang P. Formulation and characterization of curcuminoids loaded solid lipid nanoparticles. Int J Pharm. 2007;337(1-2):299-306.

98. Anand P, Nair HB, Sung B, et al. Design of curcumin-loaded PLGA nanoparticles formulation with enhanced cellular uptake, and increased bioactivity in vitro and superior bioavailability in vivo. Biochem Pharmacol. 2010;79(3):330-338.

99. Dhule SS, Penfornis P, Frazier T, et al. Curcumin-loaded $\gamma$-cyclodextrin liposomal nanoparticles as delivery vehicles for osteosarcoma. Nanomedicine. 2012;8(4):440-451.

100. Kundu P, Mohanty C, Sahoo SK. Antiglioma activity of curcuminloaded lipid nanoparticles and its enhanced bioavailability in brain tissue for effective glioblastoma therapy. Acta Biomater. 2012;8(7): 2670-2687.

101. Liu J, Xu L, Liu C, et al. Preparation and characterization of cationic curcumin nanoparticles for improvement of cellular uptake. Carbohydr Polym. 2012;90(1):16-22.

102. Barenholz Y. Liposome application: problems and prospects. Curr Opin Colloid Interface Sci. 2001;6(1):66-77.
103. Nishiyama N, Kataoka K. Current state, achievements, and future prospects of polymeric micelles as nanocarriers for drug and gene delivery. Pharmacol Ther. 2006;112(3):630-648.

104. Bellott R, Pouna P, Robert J. Separation and determination of liposomal and non-liposomal daunorubicin from the plasma of patients treated with Daunoxome. J Chromatogr B Biomed Sci Appl. 2001;757(2): 257-267.

105. Junghanns JU, Müller RH. Nanocrystal technology, drug delivery and clinical applications. Int J Nanomedicine. 2008;3(3):295-309.

106. Nijhara R, Balakrishnan K. Bringing nanomedicines to market: regulatory challenges, opportunities, and uncertainties. Nanomedicine. 2006;2(2):127-136.

107. Hawkins MJ, Soon-Shiong P, Desai N. Protein nanoparticles as drug carriers in clinical medicine. Adv Drug Deliv Rev. 2008;60(8): 876-885.

108. Kratz F. Albumin as a drug carrier: design of prodrugs, drug conjugates and nanoparticles. J Control Release. 2008;132(3):171-183.

109. Nakanishi T, Fukushima S, Okamoto K, et al. Development of the polymer micelle carrier system for doxorubicin. J Control Release. 2001;74(1-3):295-302.

110. Tsukioka Y, Matsumura Y, Hamaguchi T, Koike H, Moriyasu F, Kakizoe T. Pharmaceutical and biomedical differences between micellar doxorubicin (NK911) and liposomal doxorubicin (Doxil). Jpn J Cancer Res. 2002;93(10):1145-1153.

111. Shea JE, Nam KH, Rapoport N, Scaife CL. Genexol inhibits primary tumour growth and metastases in gemcitabine-resistant pancreatic ductal adenocarcinoma. HPB (Oxford). 2011;13(3):153-157.

112. Alakhov V, Klinski E, Li SM, et al. Block copolymer-based formulation of doxorubicin. From cell screen to clinical trials. Colloids Surf B Biointerfaces. 1999;16(1-4):113-134.

113. Danson S, Ferry D, Alakhov V, et al. Phase I dose escalation and pharmacokinetic study of pluronic polymer-bound doxorubicin (SP1049C) in patients with advanced cancer. Br J Cancer. 2004;90(11):2085-2091.

114. Bogunia-Kubik K, Sugisaka M. From molecular biology to nanotechnology and nanomedicine. Biosystems. 2002;65(2-3):123-138.

115. Fattal E, Bochot A. State of the art and perspectives for the delivery of antisense oligonucleotides and siRNA by polymeric nanocarriers. Int J Pharm. 2008;364(2):237-248.

116. Nafee N, Taetz S, Schneider M, Schaefer UF, Lehr CM. Chitosancoated PLGA nanoparticles for DNA/RNA delivery: effect of the formulation parameters on complexation and transfection of antisense oligonucleotides. Nanomedicine. 2007;3(3):173-183.

117. Zhong J, Yao X, Li DL, et al. Large scale preparation of midkine antisense oligonucleotides nanoliposomes by a cross-flow injection technique combined with ultrafiltration and high-pressure extrusion procedures. Int J Pharm. 2013;441(1-2):712-720.

118. Sioss JA, Bhiladvala RB, Pan W, et al. Nanoresonator chip-based RNA sensor strategy for detection of circulating tumor cells: response using PCA3 as a prostate cancer marker. Nanomedicine. 2012;8(6):1017-1025

119. Veiseh O, Gunn JW, Zhang M. Design and fabrication of magnetic nanoparticles for targeted drug delivery and imaging. Adv Drug Deliv Rev. 2010;62(3):284-304.

120. Mura S, Couvreur P. Nanotheranostics for personalized medicine. $A d v$ Drug Deliv Rev. 2012;64(13):1394-1416.

121. Shiraishi K, Endoh R, Furuhata H, et al. A facile preparation method of a PFC-containing nano-sized emulsion for theranostics of solid tumors. Int J Pharm. 2011;421(2):379-387.

122. Shalviri A, Foltz WD, Cai P, Rauth AM, Wu XY. Multifunctional terpolymeric MRI contrast agent with superior signal enhancement in blood and tumor. J Control Release. 2013;167(1):11-20.

123. He Q, Ma M, Wei C, Shi J. Mesoporous carbon@silicon-silica nanotheranostics for synchronous delivery of insoluble drugs and luminescence imaging. Biomaterials. 2012;33(17):4392-4402.

124. Howell M, Mallela J, Wang C, et al. Manganese-loaded lipid-micellar theranostics for simultaneous drug and gene delivery to lungs. J Control Release. 2013;167(2):210-218. 
125. Mahmoudi M, Sant S, Wang B, Laurent S, Sen T. Superparamagnetic iron oxide nanoparticles (SPIONs): development, surface modification and applications in chemotherapy. Adv Drug Deliv Rev. 2011;63(1-2):24-46.

126. Tsay JM, Trzoss M, Shi L, et al. Singlet oxygen production by Peptidecoated quantum dot-photosensitizer conjugates. J Am Chem Soc. 2007;129(21):6865-6871.

127. Spyratou E, Makropoulou M, Mourelatou EA, Demetzos C. Biophotonic techniques for manipulation and characterization of drug delivery nanosystems in cancer therapy. Cancer Lett. 2012;327(1-2): 111-122.

128. Allison RR, Mota HC, Bagnato VS, Sibata CH. Bio-nanotechnology and photodynamic therapy - state of the art review. Photodiagnosis Photodyn Ther. 2008;5(1):19-28.

129. García-Díaz M, Kawakubo M, Mroz P, et al. Cellular and vascular effects of the photodynamic agent temocene are modulated by the delivery vehicle. J Control Release. 2012;162(2):355-363.

130. Ling D, Bae BC, Park W, Na K. Photodynamic efficacy of photosensitizers under an attenuated light dose via lipid nano-carrier-mediated nuclear targeting. Biomaterials. 2012;33(21):5478-5486.
131. Sobolev AS, Jans DA, Rosenkranz AA. Targeted intracellular delivery of photosensitizers. Prog Biophys Mol Biol. 2000;73(1):51-90.

132. Roy I, Ohulchanskyy TY, Pudavar HE, et al. Ceramic-based nanoparticles entrapping water-insoluble photosensitizing anticancer drugs: a novel drug-carrier system for photodynamic therapy. J Am Chem Soc. 2003;125(26):7860-7865.

133. Kiesslich T, Berlanda J, Plaetzer K, Krammer B, Berr F. Comparative characterization of the efficiency and cellular pharmacokinetics of Foscan- and Foslip-based photodynamic treatment in human biliary tract cancer cell lines. Photochem Photobiol Sci. 2007;6(6):619-627.

134. Sibata MN, Tedesco AC, Marchetti JM. Photophysicals and photochemicals studies of zinc(II) phthalocyanine in long time circulation micelles for photodynamic therapy use. Eur J Pharm Sci. 2004;23(2):131-138.

135. Khdair A, Handa H, Mao G, Panyam J. Nanoparticle-mediated combination chemotherapy and photodynamic therapy overcomes tumor drug resistance in vitro. Eur J Pharm Biopharm. 2009;71(2):214-222.

136. Ohulchanskyy TY, Roy I, Goswami LN, et al. Organically modified silica nanoparticles with covalently incorporated photosensitizer for photodynamic therapy of cancer. Nano Lett. 2007;7(9): $2835-2842$.
International Journal of Nanomedicine

\section{Publish your work in this journal}

The International Journal of Nanomedicine is an international, peerreviewed journal focusing on the application of nanotechnology in diagnostics, therapeutics, and drug delivery systems throughout the biomedical field. This journal is indexed on PubMed Central, MedLine, CAS, SciSearch $₫$, Current Contents $₫ /$ Clinical Medicine,

\section{Dovepress}

Journal Citation Reports/Science Edition, EMBase, Scopus and the Elsevier Bibliographic databases. The manuscript management system is completely online and includes a very quick and fair peer-review system, which is all easy to use. Visit http://www.dovepress.com/ testimonials.php to read real quotes from published authors. 